\title{
Estimating Portfolio Risk for Tail Risk Protection Strategies*
}

\author{
David Happersberger ${ }^{\dagger a, b}$ Harald Lohre ${ }^{\mathrm{a}, \mathrm{b}}$ Ingmar Nolte ${ }^{\mathrm{a}}$ \\ ${ }^{\mathrm{a}}$ Centre for Financial Econometrics, Asset Markets and Macroeconomic Policy, \\ Lancaster University Management School, United Kingdom \\ ${ }^{\mathrm{b}}$ Invesco Quantitative Strategies, Frankfurt am Main, Germany
}

This version: November 27, 2019

\begin{abstract}
We forecast portfolio risk for managing dynamic tail risk protection strategies, based on extreme value theory, expectile regression, Copula-GARCH and dynamic GAS models. Utilizing a loss function that overcomes the lack of elicitability for Expected Shortfall, we propose a novel Expected Shortfall (and Value-at-Risk) forecast combination approach, which dominates simple and sophisticated standalone models as well as a simple average combination approach in modelling the tail of the portfolio return distribution. While the associated dynamic risk targeting or portfolio insurance strategies provide effective downside protection, the latter strategies suffer less from inferior risk forecasts given the defensive portfolio insurance mechanics.
\end{abstract}

JEL classification: C13, C14, C22, C53, G11.

Keywords: Tail Risk Protection, CPPI, DPPI, Risk Modelling, Value-at-Risk, Expected Shortfall, Forecast Combination, Return Synchronization.

${ }^{*}$ We thank the editor (John A. Doukas), two anonymous referees, Torben Andersen, Ole Christian Bech-Moen, Christian Groll, Manel Kammoun, Mark Kritzman, Yifan Li, Stefan Mittnik, James Taylor, Ralf Wilke as well as the participants of the 2017 Paris Financial Management Conference (PFMC), the 2017 CEQURA Conference on Advances in Financial and Insurance Risk Management in Munich, the 3rd KoLa Workshop on Finance and Econometrics at Lancaster University Managament School in 2017, the 2017 Doctoral Workshop on Applied Econometrics at the University of Strasbourg, the 2017 Global Research Meeting of Invesco Quantitative Strategies in Boston, the 2018 Frontiers of Factor Investing Conference in Lancaster, the 2018 FMA European Conference in Kristiansand and the 2018 IFABS Porto Conference for fruitful discussions and suggestions. This work was supported by funding from the Economic and Social Research Council (UK). Note that this paper expresses the authors' views that do not have to coincide with those of Invesco.

${ }^{\dagger}$ Corresponding author. Email: d.happersberger@lancaster.ac.uk. 


\section{Introduction}

Tail risk hedging strategies are of vital interest to many market participants to protect investment portfolios against extreme negative market moves. An obvious way of protection is the purchase of a put option. However, such a strategy can be expensive, since the option premium is payable each investment period, although the protection could prove unnecessary in the majority of periods. A possible alternative are dynamic asset allocation strategies, which aim to improve the downside risk profile of an investment strategy without jeopardizing its long-term return potential by dynamically shifting between a risky asset (or portfolio) and a risk-free asset.

Risk targeting strategies ${ }^{1}$ are one such possibility (Hocquard, $\mathrm{Ng}$, and Papageorgiou, 2013; Perchet, De Carvalho, Heckel, and Moulin, 2015; Bollerslev, Hood, Huss, and Pedersen, 2018). A risk targeting strategy controls portfolio risk over time by taking advantage of the negative relationship between risk and return. Specifically, the investment exposure of the portfolio is adjusted according to updated risk forecasts in order to keep the ex-ante risk at a constant target level. A stricter way to limit downside risk is to apply portfolio insurance strategies, such as the constant proportion portfolio insurance (CPPI) strategy (Perold, 1986; Black and Jones, 1987, 1988; Perold and Sharpe, 1988). Herein, the investor defines a minimum capital level to be preserved at the end of the investment period. The key element to determine the investment exposure to the risky asset is the so-called multiplier. It represents the inverse of the maximum sudden loss of the risky asset, so that a given risk budget will not be fully consumed and the portfolio value will not fall below the protection level. Initially, the multiplier was assumed to be static and unconditional (e.g. Bertrand and Prigent, 2002; Balder, Brandl, and Mahayni, 2009; Cont and Tankov, 2009). However, given the empirical characteristics of financial assets, such as time-varying volatility or volatility clustering (cf. Longin and Solnik, 1995; Andersen, Bollerslev, Christoffersen, and Diebold, 2006), other studies (e.g. Hamidi, Maillet, and Prigent, 2014) propose to model the multiplier as time-varying and conditional. The corresponding strategy is known as dynamic proportion portfolio insurance (DPPI). ${ }^{2}$

The success of both dynamic tail risk protection strategies strongly depends on the success of forecasting tail risk (Perchet, De Carvalho, Heckel, and Moulin, 2015). Given a plethora of available risk models, we contribute to the existing literature on tail risk protection strategies by investigating suitable risk models for timely managing the investment exposure in dynamic tail risk protection strategies. At the same time, we contribute to the literature on risk model evaluation by not only assessing the statistical performance but also its economical relevance when testing the risk forecasts in a relevant portfolio application.

Risk targeting strategies have been extensively backtested using historical data, and are known to show superior performance compared to a simple buy-and-hold strategy (Cooper, 2010; Kirby and Ostdiek, 2012; Ilmanen and Kizer, 2012; Giese, 2012). Hallerbach (2012, 2015) demonstrates that the

\footnotetext{
${ }^{1}$ Risk targeting strategies are also known as constant risk, target risk or inverse risk weighting strategies.

${ }^{2}$ For a comprehensive literature review on portfolio insurance and CPPI/DPPI multipliers, see Benninga (1990); Black and Perold (1992); Basak (2002); Dichtl and Drobetz (2011); Hamidi, Maillet, and Prigent (2014), among others.
} 
Sharpe ratio increases, even if the portfolio mean return is constant over time. Not only do constant volatility portfolios deliver higher Sharpe ratios than their passive counterpart but they also reduce drawdowns (Hocquard, Ng, and Papageorgiou, 2013). Similar to the risk targeting strategy that we apply, the dynamic VaR portfolio insurance strategy of Jiang, Ma, and An (2009) aims at controlling the exposure of a risky asset such that a specified Value-at-Risk is not violated. However, their strategy can only be applied to parametric location-scale models, while the one we apply is compatible with any type of risk model. In a similar vein, Bollerslev, Hood, Huss, and Pedersen (2018) use a risk targeting strategy to compare realized volatility models to more conventional procedures that do not incorporate the information in high-frequency intraday data.

The literature on dynamic proportion portfolio insurance puts forward various ways to model the conditional time-varying multiplier. While Ben Ameur and Prigent $(2007,2014)$ employ GARCH-type models, Hamidi, Jurczenko, and Maillet (2009) and Hamidi, Maillet, and Prigent (2009) define the multiplier as a function of a dynamic autoregressive quantile model of the Value-at-Risk according to Engle and Manganelli (2004). In contrast, Chen, Chang, Hou, and Lin (2008) propose a multiplier framework based on genetic programming. More recently, Hamidi, Maillet, and Prigent (2014) employ a dynamic autoregressive expectile (DARE) model to estimate the conditional multiplier. ${ }^{3}$ All these papers provide evidence that the DPPI strategy, based on a time-varying conditional risk estimate, outperforms the traditional CPPI strategy.

We are particularly interested in comparing different ways to determine the risky investment exposure of dynamic tail risk protection strategies, assessing various models to estimate a portfolio's downside risk measured by Value-at-Risk (VaR) and Expected Shortfall (ES). While the literature suggests a myriad of VaR and ES models - Andersen, Bollerslev, Christoffersen, and Diebold (2006, 2013), Kuester, Mittnik, and Paolella (2006), and Righi and Ceretta (2015) provide thorough summaries on market risk modelling - practitioners still only use a limited number of them. This discrepancy might be due to complexity, (computational) efficiency or the perception that the incremental benefit of implementing a highly sophisticated model is minor. Therefore, we examine simple methods that are common among practitioners as well as more involved methods to predict VaR and ES. Specifically, we consider: historical simulation, Cornish-Fisher Approximation, RiskMetrics, quantile and expectile regressions, extreme value theory, Copula-GARCH and recent generalized autoregressive score (GAS) models (including one and two-factor GAS models as well as the hybrid GAS/GARCH model).

The primary issue of these (standalone) risk models is that the models' performances and reliabilities in accurately predicting risk often depend heavily on the data. While a parsimonious model can perform well in stable markets, it might fail during a volatile period. Likewise, highly parameterized models can be adequate during periods of high volatility, but might be easily outperformed by

\footnotetext{
${ }^{3}$ Hamidi, Maillet, and Prigent (2014) model the multiplier as a function of the expected shortfall determined by a combination of quantile functions in order to reduce the potential model error. Specifically, they combine the historical simulation approach, three methods based on distributional assumptions and four methods based on expectiles and conditional autoregressive specifications into the DARE approach.
} 
simpler approaches in less turbulent times (cf. Bayer, 2018). Hence, it is often beneficial to combine predictions originating from various approaches. Reviewing forecasting combinations, Timmermann (2006) provides three arguments in favor of combining forecasts to enhance the predictive performance relative to standalone models: first, there are diversification gains arising from the combination of forecasts computed from different assumptions, specifications or information sets. Second, combination forecasts tend to be robust against structural breaks. Third, the influence of potential misspecification biases and measurement errors of the individual models is reduced due to averaging over a set of forecasts derived from several models. While there exist various approaches to combine VaR predictions (see Bayer, 2018, for a summary), the literature is lacking methods that combine ES predictions. This shortage relates to the fact that ES is not "elicitable", i.e. there does not exist a loss function such that the correct ES forecast is the solution to minimizing the expected loss (cf. Gneiting, 2011). This lack of elicitability renders the estimation and backtesting of ES challenging (see Acerbi and Szekely, 2014; Embrechts and Hofert, 2014; Emmer, Kratz, and Tasche, 2015). As a remedy, Fissler and Ziegel (2016) recently introduced a class of loss functions that overcomes the lack of elicitability for ES by jointly modelling ES and VaR. Drawing on their results, we propose a novel ES (and VaR) forecast combination approach. ${ }^{4}$

Based on a global multi-asset data set consisting of stock, bond, commodity and currency indices, our empirical study documents a clear superiority of the proposed forecast combination approach over both sophisticated and more naive standalone models using a state-of-art ES and VaR backtesting framework (Kupiec, 1995; Christoffersen, 1998; McNeil and Frey, 2000; Christoffersen and Pelletier, 2004; Engle and Manganelli, 2004; Berkowitz, Christoffersen, and Pelletier, 2011; Nolde and Ziegel, 2017; Bayer and Dimitriadis, 2018; Patton, Ziegel, and Chen, 2019). In the combination of ES (and VaR) forecasts, complexity seems to actually pay off as the proposed forecast combination approach outperforms a simple average forecast. Among the standalone models, sophisticated risk models such as the extreme value theory or the Copula-GARCH approach outperform simple approaches in terms of historical accuracy and statistical fit. When subsequently feeding the risk forecasts in the tail risk protection framework, our findings are twofold. For the risk targeting strategy, we observe a clear outperformance of the more intricate methods, confirming the results from the statistical analysis. For the DPPI strategy, we likewise show that the use of more sophisticated risk models helps to protect investors from downside risk. Yet, more naive approaches do not fall short of providing downside protection. Given that the portfolio insurance strategy automatically reduces investment exposure when approaching the protection level, the less sophisticated methods' weaknesses seem to be compensated by this second line of defense.

The remainder of the paper is structured as follows: Section 2 discusses the employed tail risk protection strategies. Section 3 briefly presents the different models to estimate portfolio risk, including the novel forecast combination technique based on Fissler-Ziegel loss functions. In Section

\footnotetext{
${ }^{4}$ In recent independent work, Taylor (2019a) uses the same class of loss functions to combine VaR and ES predictions. While his methods for combining forecasts are in the spirit of Bates and Granger (1969) and Shan and Yang (2009), our methodology rather follows the approach of Halbleib and Pohlmeier (2012) and Bayer (2018).
} 
4, we perform the empirical study using a global multi-asset data set to compare the performance of dynamic tail risk protection strategies based on the different risk models. Section 5 concludes.

\section{Tail Risk Protection Strategies}

We consider a risk-averse investor who aims to limit the downside risk of his investment over an investment horizon of $H$ time steps. Further, let $t=1,2, \ldots, T$ be the time index of portfolio rebalancing and $I(t)=t-\left(\left\lceil\frac{t}{H}\right\rceil-1\right) H$ a subindex for each investment period $\left\lceil\frac{t}{H}\right\rceil$, so that the latter runs from 1 to $H$ in each investment period. At the beginning of each investment period $\left\lceil\frac{t}{H}\right\rceil$, i.e. at $I(t)=1$, the investor determines a risk target that should be achieved at the end of the period, i.e. at $I(t)=H$. The management of the protected portfolio follows a dynamic portfolio allocation. In particular, the value of the protected portfolio, denoted by $V_{t}$, is invested in a risky asset (or portfolio) and a non-risky asset in such a way that the given risk target will not be violated. The price of the risky asset at time $t$ is denoted as $P_{t}$ so that the logarithmic return from $t$ to $t+1$ is $r_{t+1}=\log \left(P_{t+1} / P_{t}\right)$. Accordingly, the price and return of the non-risky asset is denoted as $B_{t}$ and $r_{f, t}$. To explicitly determine the exposure to the risky asset $e_{t}$, we need to forecast the downside risk of the risky asset over the next day, quantified by a risk measure $\rho(\cdot)$.

\subsection{Risk Targeting Strategies}

A risk targeting strategy systematically adjusts exposure to a given asset (or portfolio) conditional on its current risk (forecast) in order to maintain a pre-specified target risk level. Specifically, if a portfolio's current risk is higher than the target level, one would lower the investment exposure by shifting towards the risk-free asset, and vice versa if the current risk is lower than the target level. The rationale for maintaining a constant risk level is twofold (see Hocquard, Ng, and Papageorgiou, 2013). First, most significant market corrections have been preceded by an increase in risk. By conditioning their exposure on market risk, investors can dampen the impact of a market correction. Second, empirical evidence suggests that asset returns tend to be greater during periods of low risk. Consequently, investors should maximize asset exposure during these periods, taking advantage of a favorable risk-reward tradeoff. Conversely, they should decrease asset exposure when risk increases to maintain the desired risk level.

Given the level of ex-ante risk of the underlying risky asset $\rho_{t}\left(r_{t+1}\right)$ and the predefined target risk $\bar{\rho}$, the allocation to the risky asset $e_{t}$ is simply $\bar{\rho} / \rho_{t}\left(r_{t+1}\right)$. As $\rho_{t}\left(r_{t+1}\right)$ is unknown, we utilize a forecast based on the information available at time $t, \mathcal{F}_{t}$ :

$$
e_{t} \equiv \frac{\bar{\rho}}{\hat{\rho}_{t}\left(r_{t+1} \mid \mathcal{F}_{t}\right)} .
$$

Correspondingly, the weight of the risk-free asset is given by $1-e_{t}$. 


\subsection{Constant and Dynamic Proportion Portfolio Insurance}

The constant proportion portfolio insurance (CPPI) strategy (see Perold, 1986; Black and Jones, 1987, 1988; Perold and Sharpe, 1988) dynamically shifts between the risky and non-risky asset to guarantee the investor to at least recover a given proportion of its initial capital. At the beginning of each investment period $\left\lceil\frac{t}{H}\right\rceil$, i.e. at $I(t)=1$, the investor determines this minimum portfolio value, or floor $F_{\left\lceil\frac{t}{H}\right\rceil}$, that should be preserved at the end of the period, i.e. at $I(t)=H$. The corresponding risk capital, called the cushion, is derived as the difference of portfolio value, $V_{t}$, and discounted floor (i.e. the net present value, $\operatorname{NPV}(\cdot)$, of the floor):

$$
C_{t}=V_{t}-\mathrm{NPV}_{t}\left(F_{\left\lceil\frac{t}{H}\right\rceil}\right) .
$$

The cushion represents a certain amount of the portfolio value to absorb potential market shocks before the portfolio manager can rebalance the portfolio. In order to avoid a breach of the discounted floor, the investment exposure to the risky asset, defined as $E_{t}=e_{t} V_{t}$, should be set such that the cushion at $t$ is at least as high as the maximum sudden drop in the portfolio value between the rebalancing dates $t$ and $t+1$, i.e.

$$
C_{t} \geq V_{t}\left|\inf \left(\log \left(\frac{V_{t+1}}{V_{t}}\right)\right)\right| .
$$

As the portfolio consists of the risky and the non-risky asset, (3) can be simplified to

$$
C_{t} \geq e_{t} V_{t}\left|\inf \left(\log \left(\frac{P_{t+1}}{P_{t}}\right)\right)\right| \text {. }
$$

Rearranging (4) then yields the (total) exposure to the risky asset as

$$
E_{t} \leq C_{t}\left|\inf \left(r_{t+1}\right)\right|^{-1}=C_{t} m
$$

where $m \equiv\left|\inf \left(r_{t+1}\right)\right|^{-1}$ is the multiplier. ${ }^{5}$ The multiplier indicates how often a given cushion can be invested in the risky asset without breaching the floor. Thus, it reflects the investor's risk tolerance. The higher the multiplier, the more the investor will participate in upward market movements of the underlying. But the higher the multiplier, the faster the portfolio will reach the floor when there is a sustained decrease in the underlying's price. In order to allow for the highest possible participation in the underlying risky asset, it is common to set $E_{t}$ such that equality holds in (5). The remainder is invested in the risk-free asset.

If rebalancing were continuous and price movements sufficiently smooth, the CPPI allocation rule would ensure that the portfolio does not fall below the floor (Cont and Tankov, 2009; Balder, Brandl, and Mahayni, 2009; Hamidi, Hurlin, Kouontchou, and Maillet, 2015; Ardia, Boudt, and Wauters, 2016). However, with discrete rebalancing and jumps in prices, there is a non-negligible probability

\footnotetext{
${ }^{5}$ We follow Benninga (1990) and restrict the participation ratio to vary between $0 \%$ and $100 \%$ of the risky asset in order to rule out short positions. This approach leads to a slightly different exposure definition: $E_{t}=\max \left[\min \left(C_{t} m, V_{t}\right), 0\right]$.
} 
that the floor is breached. This risk of losing more than the cushion between two rebalancing dates and thus failing to ensure the protection at the end of the investment period is called gap risk. A common way to minimize gap risk is to employ the minimum return of the risky asset over the sample history, i.e. $\inf \left(r_{t+1}\right)=\min \left(r_{1}, \ldots, r_{t+1}\right)$. Then, the CPPI strategy is based on a static unconditional multiplier - often reflecting a constant worst-case scenario. Although such a conservative stance would have meaningfully addressed catastrophic drawdowns during extreme market turmoil, it would also have unduly capped upside potential over the long term. Dynamic proportion portfolio insurance (DPPI) is designed to introduce more flexibility. Instead of using a static multiplier, the risk budget adapts dynamically to changes in a risk forecast, measured by $\hat{\rho}(\cdot)$. Thus, the exposure changes to

$$
E_{t}=C_{t}\left|\hat{\rho}_{t}\left(r_{t+1} \mid \mathcal{F}_{t}\right)\right|^{-1}=C_{t} m_{t}
$$

where the risk forecast $\hat{\rho}_{t+1}$ is based on information $\mathcal{F}_{t}$ and measures the risk when the risky asset price $S$ evolves from $t$ to $t+1$. The dynamic multiplier is therefore given by

$$
m_{t}=\left|\hat{\rho}_{t}\left(r_{t+1} \mid \mathcal{F}_{t}\right)\right|^{-1} .
$$

In this way, the portfolio's exposure to the risky asset reacts to changes in the risk forecast - ensuring that it does not remain artificially low as a result of a constant conservative risk assumption. For this to work in practice, the risk model must be capable of quickly homing in on volatility spikes, and just as quickly readjusting to a normalization of market volatility.

The advantage of the CPPI and DPPI strategy, respectively, is the simple practical implementation that does not call for forecasting the returns of the risky asset. Major drawbacks are the strategies' path dependencies as well as the lock-in effect. Depending on the underlying portfolio return path, the CPPI/DPPI strategy can deliver a wide range of outcomes. In general, the more volatile the risky asset, the lower the average participation ratio. While the CPPI strategy is fully exposed to the problem of path dependency, the DPPI strategy can mitigate this problem at least to some extent by quickly reacting to market changes. The lock-in effect occurs when the cushion is entirely consumed by portfolio losses. The CPPI/DPPI strategy is then fully invested in the risk-free asset until the end of the investment period and no participation in subsequent upward movements is possible.

\section{Portfolio Risk Modelling}

Given the vast amount of available risk models (see Kuester, Mittnik, and Paolella, 2006; Nadarajah, Zhang, and Chan, 2014), we focus on a few yet distinct approaches, ranging from rather simple models that are widely used among practitioners to more intricate models that are state-of-the-art in the academic literature. We consider both portfolio-level (aggregated, "top-down") and asset-level (disaggregated, "bottom-up") risk modelling. In addition, we propose a new VaR and ES forecast combination approach based on a loss function that overcomes the lack of elicitability for ES by jointly modelling ES and VaR. Following the description of downside risk measurement, we summarize the 
various methods in this section. ${ }^{6}$

\subsection{Conditional Risk Measurement}

The literature suggests various ways to quantify market risk of financial assets. As we are particularly interested in protecting risky portfolios against extreme market losses, we resort to the most common downside risk measures, Value-at-Risk (VaR) and Expected Shortfall (ES). VaR measures the maximum potential portfolio loss at a given confidence level. ${ }^{7}$ Therefore, the VaR forecast from $t$ to $t+1$ is simply the negative $p$-quantile of the conditional return distribution at $t+1$, that is,

$$
\operatorname{VaR}_{t+1 \mid t}^{p}=Q_{p}\left(r_{t+1} \mid \mathcal{F}_{t}\right)=\inf _{x}\left\{x \in \mathbb{R}: P\left(r_{t+1} \leq x \mid \mathcal{F}_{t}\right) \geq p\right\}
$$

where $p \in(0,1)$ is the probability level, $Q_{p}(\cdot)$ denotes the quantile function, $r_{t}$ reflects the return of the asset (portfolio) in period $t$ and $\mathcal{F}_{t}$ represents the information available at time $t$.

Although VaR is still the risk measure of choice in the financial industry, it has been criticized for disregarding outcomes beyond the specified VaR-quantile. Moreover, VaR is not a subadditive risk measure. This property posits that the total portfolio risk should not be greater than the sum of the risks of its constituents (see Artzner, Delbaen, Eber, and Heath, 1999; Acerbi and Tasche, 2002; Taylor, 2008). Expected shortfall, also known as conditional VaR or expected tail loss, is a risk measure that overcomes these weaknesses by aggregating information about the tail of the portfolio return distribution. It is defined as the conditional expectation of the return given that VaR is exceeded (see Yamai, Yoshiba, et al., 2002), specifically

$$
\mathrm{ES}_{t+1 \mid t}^{p}=p^{-1} \int_{0}^{p} \operatorname{VaR}_{t+1 \mid t}^{s} d s
$$

Throughout the paper, we focus on the probability level $\mathrm{p}=1 \%$, taking a conservative stance for the tail risk protection strategies.

\subsection{Conditional Portfolio-Level Risk Models}

Generally, there are two classes of risk modelling, depending on the aggregation level. Portfolio-level analysis, as discussed in this section, requires only a univariate model based on aggregated portfolio returns. The latter can easily be constructed using portfolio holdings $\mathbf{w}_{t}=\left[w_{1, t}, w_{2, t}, \ldots, w_{N, t}\right]^{\prime}$ and

\footnotetext{
${ }^{6}$ For a rigorous discussion of the analyzed risk models, see Kuester, Mittnik, and Paolella (2006), Andersen, Bollerslev, Christoffersen, and Diebold (2013) and Righi and Ceretta (2015). Note that we do not impose the same estimate of the standard deviation in all location-scale risk models. We intentionally consider simple methods (such as historical simulation, Cornish-Fisher approximation and RiskMetrics) in a way that practitioners would often use them to compute VaR and ES forecasts. These models are then contrasted to more sophisticated methods popular in the academic literature, which we construct consistent with the original studies.

${ }^{7}$ The literature commonly uses low-probability terminology, hence we are speaking of a $1 \%$-VaR rather than a 99\%-VaR.
} 
the individual asset returns $\mathbf{r}_{t}=\left[r_{1, t}, r_{2, t}, \ldots, r_{N, t}\right]^{\prime}:$

$$
r_{\mathrm{PF}, t}=\sum_{i=1}^{N} w_{i, t} r_{i, t}=\mathbf{w}_{t}^{\prime} \mathbf{r}_{t}, \quad t=1,2, \ldots, T .
$$

While aggregation generally comes at the loss of information, Andersen, Bollerslev, Christoffersen, and Diebold (2013) argue that there is no reason why a parsimonious dynamic model should not be estimated for portfolio-level returns. If one is interested in the portfolio return distribution, one may model it directly rather than via aggregation based on a larger, and almost inevitably less well-specified, multivariate model. ${ }^{8}$

\subsubsection{Historical Simulation}

The simplest way to estimate VaR and ES is to use the sample quantile function based on historic return data, which is referred to as historical simulation (HS). Let $r_{\mathrm{PF},(1)} \leq r_{\mathrm{PF},(2)} \leq \ldots \leq r_{\mathrm{PF},(t)}$ denote the order statistics in ascending order corresponding to the original historical pseudo portfolio returns $r_{\mathrm{PF}, 1}, r_{\mathrm{PF}, 2}, \ldots, r_{\mathrm{PF}, t}$. Then, the HS-VaR for $t+1$ is simply the empirical $100 p$-th quantile or the $t p$-th order statistic, i.e.

$$
\operatorname{VaR}_{t+1 \mid t}^{p}=r_{\mathrm{PF},(\lceil t p\rceil)} .
$$

Correspondingly, the ES estimate for $t+1$ can be computed as

$$
\mathrm{ES}_{t+1 \mid t}^{p}=\left(\sum_{i=\lceil t p\rceil}^{t} r_{\mathrm{PF},(i)}\right)(t-\lceil t p\rceil)^{-1} .
$$

The main advantage of the HS approach is its computational simplicity and non-parametric dimension, i.e. VaR and ES do not rely on any distributional assumptions. In contrast, the HS approach cannot properly incorporate conditionality (see Andersen, Bollerslev, Christoffersen, and Diebold, 2006). ${ }^{9}$ This deficiency of the conventional HS approach is oftentimes highlighted by a clustering of VaR violations in time, reflecting a failure to properly account for persistent changes in market volatility. The only source of dynamics in the HS risk estimates is the evolving window used to construct historical pseudo portfolio returns. Nevertheless, the choice of the window size is crucial: too few observations will lead to sampling error, whereas too many observations will slow down the reaction to changes in the true return distribution. Moreover, the risk estimates can jump when large negative returns either enter or exit the estimation window.

\footnotetext{
${ }^{8}$ In contrast to Andersen, Bollerslev, Christoffersen, and Diebold (2013), some studies (e.g. Santos, Nogales, and Ruiz, 2012) take an opposite view regarding the validity of aggregation at portfolio level when estimating portfolio risk. They argue that univariate models are often misspecified if the true return generating process is multivariate.

${ }^{9}$ For a rigorous discussion of several serious issues of the HS approach, see Pritsker (2006).
} 


\subsubsection{Cornish-Fisher Approximation}

Another simple approach is the Cornish-Fisher Approximation (CFA) method (Zangari, 1996), where the VaR is modelled as a Taylor-series type expansion (cf. Cornish and Fisher, 1938) around the VaR of a normal distribution. Specifically, the CFA-VaR is an extension of the normal quantile function by accounting for skewness $\gamma$ and kurtosis $\delta$, and is calculated as

$$
\operatorname{VaR}_{t+1 \mid t}^{p}=\mu_{t}+\sigma_{t} F_{C F}^{-1}(p)
$$

where

$$
F_{C F}^{-1}(p) \equiv \Phi_{p}^{-1}+\left(\left[\Phi_{p}^{-1}\right]^{2}-1\right) \frac{\gamma}{6}+\left(\left[\Phi_{p}^{-1}\right]^{3}-3 \Phi_{p}^{-1}\right) \frac{\delta-3}{24}-\left(2\left[\Phi_{p}^{-1}\right]^{3}-5 \Phi_{p}^{-1}\right) \frac{\gamma^{2}}{36}
$$

and $\Phi(\cdot)$ is the standard normal cdf. Moreover, $\mu_{t}$ and $\sigma_{t}$ are computed by the sample mean and sample standard deviation, respectively.

Boudt, Peterson, and Croux (2008) show how the Edgeworth and Cornish-Fisher expansions of the density and quantile functions can be used to obtain an estimator for ES that delivers accurate downside risk estimates even in the presence of non-normal returns. The modified or Cornish-Fisher ES is thus computed as

$$
\mathrm{ES}_{t+1 \mid t}^{p}=\mu_{t}+\sigma_{t} \mathbb{E}_{F_{C F}}\left[z \mid z \leq F_{C F}^{-1}(p)\right]
$$

where

$$
\begin{aligned}
\mathbb{E}_{F_{C F}}\left[z \mid z \leq F_{C F}^{-1}(p)\right]= & -\frac{1}{p}\left(\phi\left(F_{C F}^{-1}(p)\right)+\frac{\delta}{24}\left[I^{4}-6 I^{2}+3 \phi\left(F_{C F}^{-1}(p)\right)\right]+\frac{\gamma}{6}\left[I^{3}-3 I^{1}\right]\right. \\
& \left.+\frac{\gamma^{2}}{72}\left[I^{6}-15 I^{4}+45 I^{2}-15 \phi\left(F_{C F}^{-1}(p)\right)\right]\right)
\end{aligned}
$$

with

$$
I^{q}= \begin{cases}\sum_{i=1}^{q / 2}\left(\frac{\prod_{j=1}^{q / 2} 2 j}{\prod_{j=1}^{i} 2 j}\right) g_{p}^{2 i} \phi\left(g_{p}\right)+\left(\prod_{j=1}^{q / 2} 2 j\right) \phi\left(g_{p}\right) & \text { for } q \text { even } \\ \sum_{i=0}^{q^{*}}\left(\frac{\prod_{j=0}^{q^{*}}(2 j+1)}{\prod_{j=0}^{i}(2 j+1)}\right) g_{p}^{2 i+1} \phi\left(g_{p}\right)-\left(\prod_{j=0}^{q^{*}} 2(j+1)\right) \phi\left(g_{p}\right) & \text { for } q \text { odd }\end{cases}
$$

and $q^{*}=(q-1) / 2, g_{p}=F_{C F}^{-1}(p) . \phi(\cdot)$ denotes the standard normal pdf.

The main advantage of the CFA method is its ability to account for fat tails. However, the CFA-VaR is not necessarily monotone, i.e. the $1 \%$-VaR might be smaller than the $5 \%$-VaR. Martin and Arora (2017) also document that the CFA-VaR and CFA-ES suffer in terms of statistical efficiency.

\subsubsection{Quantile/Expectile Regression}

As VaR and ES are directly linked to quantiles and expectiles, a natural approach to risk modelling employs quantile and expectile regressions. The basic idea of quantile regression is to directly model 
the conditional quantile rather than the whole distribution of portfolio returns. More precisely, the conditional $p$-quantile, $Q_{p}\left(r_{P F, t} \mid \mathcal{F}_{t-1}\right)=-\mathrm{VaR}_{t \mid t-1}$, is modelled as a parametric function of the information $\mathcal{F}_{t-1}$ :

$$
\operatorname{VaR}_{t \mid t-1}^{p} \equiv g_{p}\left(\mathcal{F}_{t-1} ; \beta_{p}\right)
$$

where $g(\cdot, \cdot)$ and the parameter vector $\beta$ explicitly depend on $p$. Following Koenker and Bassett (1978), the conditional sample $p$-quantile can be found as the solution to

$$
\min _{\beta_{p}}\left\{\sum_{r_{\mathrm{PF}, t} \geq \mathrm{VaR}_{t \mid t-1}^{p}} p\left|r_{\mathrm{PF}, t}-\mathrm{VaR}_{t \mid t-1}^{p}\right|+\sum_{r_{\mathrm{PF}, t}<\mathrm{VaR}_{t \mid t-1}^{p}}(1-p)\left|r_{\mathrm{PF}, t}-\mathrm{VaR}_{t \mid t-1}^{p}\right|\right\}
$$

where we determine $\operatorname{VaR}_{t}^{p}$ by the conditional autoregressive Value-at-Risk (CAViaR) specification of Engle and Manganelli (2004). In particular, we adopt their asymmetric slope CAViaR model ${ }^{10}$ that $^{2}$ is given by

$$
\operatorname{VaR}_{t \mid t-1}^{p}=\beta_{0}+\beta_{1} \operatorname{VaR}_{t-1 \mid t-2}^{p}+\beta_{2} \max \left[r_{\mathrm{PF}, t-1}, 0\right]+\beta_{3} \max \left[-r_{\mathrm{PF}, t-1}, 0\right] .
$$

In a similar fashion, we can use expectile regression to estimate ES. In particular, we employ the conditional autoregressive expectile (CARE) model of Taylor (2008). First, we consider that the population $\tau_{p}$ expectile of $r_{\mathrm{PF}, t}$ is the parameter $\mu_{\tau_{p}}$ that minimizes the function $\mathbb{E}\left[\left|\tau_{p}-\mathbb{1}\left(r_{\mathrm{PF}, t}-\mu_{\tau_{p}}\right)\right|\left(r_{\mathrm{PF}, t}-\mu_{\tau_{p}}\right)^{2}\right]$. Hence, we can represent the conditional expectile as a parametric function of past information, i.e. $\mu_{\tau_{p}}\left(r_{\mathrm{PF}, t}\right) \equiv h_{\tau_{p}}\left(\mathcal{F}_{t-1} ; \gamma_{\tau_{p}}\right)$. The parameters $\gamma_{\tau_{p}}$ can be estimated using asymmetric least squares (cf. Newey and Powell, 1987), i.e.

$$
\min _{\gamma_{\tau_{p}}}\left\{\sum_{r_{\mathrm{PF}, t}}\left|\tau_{p}-\mathbb{1}\left(r_{\mathrm{PF}, t}<h_{\tau_{p}}\left(\mathcal{F}_{t-1} ; \gamma_{\tau_{p}}\right)\right)\right|\left(r_{\mathrm{PF}, t}-h_{\tau_{p}}\left(\mathcal{F}_{t-1} ; \gamma_{\tau_{p}}\right)\right)^{2}\right\},
$$

where $\mathbb{1}(\cdot)$ denotes the indicator function. Similar to the asymmetric slope CAViaR model, we assume the conditional expectile to have an asymmetric slope specification. The ES can then be computed as the product of a correction term and the conditional expectile, i.e.

$$
\mathrm{ES}_{t \mid t-1}^{p}=\left(1+\frac{\tau_{p}}{\left(1-2 \tau_{p}\right) p}\right)\left(\gamma_{0}+\gamma_{1} \mu_{\tau_{p}}\left(r_{\mathrm{PF}, t-1}\right)+\gamma_{2} \max \left[r_{\mathrm{PF}, t-1}, 0\right]+\beta_{3} \max \left[-r_{\mathrm{PF}, t-1}, 0\right]\right) .
$$

The quantile and expectile regression approach are charming because no explicit distributional assumption for the time series behaviour of returns is needed, thus reducing the risk of model misspecification. The main drawback of the CAViaR modelling strategy is that it might generate outof-order quantiles similar to the CFA method. Also, estimation of model parameters is challenging. ${ }^{11}$

\footnotetext{
${ }^{10}$ For the sake of simplicity, we focus on one CAViaR model. Particularly, we choose the asymmetric slope specification because of its ability to accommodate the leverage effect.

${ }^{11}$ We thank James Taylor for providing the Gauss code for his CARE models.
} 


\subsubsection{Extreme Value Theory}

As we are primarily interested in the tails of the portfolio distribution, it seems natural to resort to extreme value theory (EVT) which estimates the tails based on extrapolating from available observations. McNeil and Frey (2000) propose a semi-parametric framework based on extreme value theory to describe the tail of the conditional distribution. The first step is to employ pseudo-maximumlikelihood fitting of $\operatorname{GARCH}(1,1)$ models to estimate conditional volatility forecasts $\hat{\sigma}_{t+1}$ (Engle, 1982; Bollerslev, 1986; Taylor, 1986). In a second step, we resort to EVT for estimating the tail of the innovation distribution of the $\operatorname{GARCH}(1,1)$ model. In particular, we use the peak-over-threshold method where a Generalized Pareto Distribution (GPD) is fitted to the negative of portfolio returns over a specified threshold. ${ }^{12}$ The quantile $\hat{z}_{p}$ can then be estimated as

$$
\hat{z}_{p}=u+\frac{\hat{\beta}}{\hat{\xi}}\left[\left(\frac{1-p}{n_{u} / n}\right)^{-\hat{\xi}}-1\right],
$$

where $\hat{\beta}$ and $\hat{\xi}$ are the GPD estimates and $n_{u}$ is the number of observations above threshold $u$. Consequently, the VaR and ES forecasts can be computed as

$$
\begin{aligned}
\operatorname{VaR}_{t+1 \mid t}^{p} & =\hat{\sigma}_{t+1} \hat{z}_{p} \\
\mathrm{ES}_{t+1 \mid t}^{p} & =\hat{\sigma}_{t+1} \hat{z}_{p}\left(\frac{1}{1-\hat{\xi}}+\frac{\hat{\beta}-\hat{\xi} u}{(1-\hat{\xi}) \hat{z}_{p}}\right) .
\end{aligned}
$$

The GARCH fitting in the first step enables us to capture certain stylized facts such as time-varying volatility, fat tails and volatility clustering. Then, EVT is particularly suitable to estimate the tails of the distribution. The crucial assumption of EVT is, however, that one is in the tail of the distribution. Hence, the difficulty is the determination of the threshold. If the threshold is too low, then the approximation can hardly be justified and the associated risk estimates may be biased. Vice versa, if the threshold is too high, there are too few observations over the threshold resulting in highly volatile estimates.

\subsubsection{GAS Models}

Patton, Ziegel, and Chen (2019) propose dynamic VaR and ES models drawing on recent results from statistical decision theory that overcome the lack of elicitability ${ }^{13}$ for ES by jointly modelling ES and VaR (cf. Fissler and Ziegel, 2016). ${ }^{14}$ These models are semi-parametric in that they impose parametric structures for the dynamics of ES and VaR according to the "generalized autoregressive

\footnotetext{
${ }^{12}$ We follow McNeil and Frey (2000) when determining the thresholds. See their paper for details.

${ }^{13}$ A statistical functional (e.g. a risk measure) is said to be "elicitable" if there exists a loss function such that the correct forecast of the functional is the solution to minimizing the expected loss (cf. Gneiting, 2011; Fissler and Ziegel, 2016; Patton, Ziegel, and Chen, 2019). For example, the mean is elicitable using the quadratic loss function, and VaR is elicitable using the piecewise-linear or "tick" loss function.

${ }^{14}$ Similar to the approach of Patton, Ziegel, and Chen (2019), Taylor (2019b) proposes using the asymmetric Laplace distribution to jointly estimate dynamic models for VaR and ES.
} 
score" (GAS) framework proposed by Creal, Koopman, and Lucas (2013) and Harvey (2013), but are completely agnostic about the conditional distribution of returns (aside from regularity conditions required for estimation and inference).

\section{Two-Factor GAS model}

The two-factor GAS $(1,1)$ model allows ES and VaR to evolve as two separate, correlated processes:

$$
\left[\begin{array}{c}
\operatorname{VaR}_{t+1 \mid t}^{p} \\
\mathrm{ES}_{t+1 \mid t}^{p}
\end{array}\right]=\mathbf{v}+\mathbf{B}\left[\begin{array}{c}
\operatorname{VaR}_{t \mid t-1}^{p} \\
\mathrm{ES}_{t \mid t-1}^{p}
\end{array}\right]+\mathbf{A H}_{t}^{-1} \nabla_{t}
$$

where $\mathbf{v}$ is a $(2 \times 1)$-vector and $\mathbf{B}$ and $\mathbf{A}$ are $(2 \times 2)$-matrices. The forcing variable in this model is a function of the derivative, $\nabla_{t}$, and the Hessian, $\mathbf{H}_{t}$, of the "FZ loss function" (see Section 3.4 for details on this loss function):

$$
\mathbf{H}_{t}^{-1} \nabla_{t}=\left[\begin{array}{c}
\frac{-1}{k_{p}} \lambda_{\mathrm{VaR}, t} \\
\frac{-1}{p}\left(\lambda_{\mathrm{VaR}, t}+p \lambda_{\mathrm{ES}, t}\right)
\end{array}\right]
$$

where $k_{p}$ is a constant with the same sign as $\operatorname{VaR}_{t}$ and

$$
\begin{aligned}
\lambda_{\mathrm{VaR}, t} & \equiv-\operatorname{VaR}_{t \mid t-1}^{p}\left(\mathbb{1}\left(r_{\mathrm{PF}, t} \leq \mathrm{VaR}_{t \mid t-1}^{p}\right)-p\right) \\
\lambda_{\mathrm{ES}, t} & \equiv \frac{1}{p} \mathbb{1}\left(r_{\mathrm{PF}, t} \leq \mathrm{VaR}_{t \mid t-1}^{p}\right) \mathrm{VaR}_{t \mid t-1}^{p}-\mathrm{ES}_{t \mid t-1}^{p} .
\end{aligned}
$$

As the second term in the model is a linear combination of the two elements of the forcing variable, and since the forcing variable is premultiplied by a coefficient matrix, say $\tilde{\mathbf{A}}$, we can equivalently use

$$
\begin{aligned}
\tilde{\mathbf{A}} \mathbf{H}_{t}^{-1} \nabla_{t} & =\mathbf{A} \boldsymbol{\lambda}_{t}, \\
\text { where } \quad \lambda_{t} & \equiv\left[\lambda_{\mathrm{VaR}, t}, \lambda_{\mathrm{ES}, t}\right]^{\prime} .
\end{aligned}
$$

\section{One-Factor GAS model}

As a simpler variant, Patton, Ziegel, and Chen (2019) introduce the one-factor GAS model, where both VaR and ES are driven only by a single variable, $\kappa_{t}$,

$$
\begin{aligned}
\operatorname{VaR}_{t+1 \mid t}^{p} & =a \exp \left(\kappa_{t+1}\right), \\
\operatorname{ES}_{t+1 \mid t}^{p} & =b \exp \left(\kappa_{t+1}\right),
\end{aligned}
$$

where $b<a<0$ and

$\kappa_{t}=\omega+\beta \kappa_{t-1}+\gamma \frac{1}{b \exp \left(\operatorname{ES}_{t-1 \mid t-2}^{p}\right)}\left(\frac{1}{p} \mathbb{1}\left(r_{\mathrm{PF}, t-1} \leq a \exp \left(\operatorname{VaR}_{t-1 \mid t-2}^{p}\right)\right) r_{\mathrm{PF}, t-1}-b \exp \left(\operatorname{ES}_{t-1 \mid t-2}^{p}\right)\right)$.

As $\omega, a$ and $b$ are not separably identifiable we set $\omega=0$. 


\section{Hybrid GAS/GARCH model}

The hybrid GAS/GARCH model of Patton, Ziegel, and Chen (2019) is a direct combination of the forcing variable suggested by a GAS structure for a one-factor model of returns, described in Eq. (31), with the successful GARCH model for volatility:

$$
r_{t+1}=\exp \left(\kappa_{t+1}\right) \eta_{t+1}, \quad \eta_{t} \sim \mathcal{F}_{\eta}(0,1)
$$

where the log-volatility $\kappa_{t}$ is specified as follows:

$$
\kappa_{t}=\omega+\beta \kappa_{t-1}+\gamma \frac{1}{\mathrm{ES}_{t-1 \mid t-2}^{p}}\left(\frac{1}{p} \mathbb{1}\left(r_{t-1} \leq \operatorname{VaR}_{t-1 \mid t-2}^{p}\right) r_{t-1}-\mathrm{ES}_{t-1 \mid t-2}^{p}\right)+\delta \log \left|r_{\mathrm{PF}, t-1}\right| .
$$

As the latent variable in this model is log-volatility, the authors use the lagged log absolute return rather than the lagged squared return, so that the units remain in line for the evolution equation for $\kappa_{t}$

Similar to quantile and expectile regressions, the semi-parametric approach of the proposed GAS models eliminates the need to specify and estimate a conditional density. While removing the possibility of a model misspecification, there might be a loss of efficiency compared with a correctly specified density model. Unlike GARCH models, the GAS models generate a smoother time series of VaR and ES estimates. While GARCH estimates are driven by lagged squared returns, and can thus be quite volatile, the GAS model estimates only use information from returns when the VaR is violated, and revert deterministically to the long-run mean on other days.

\subsection{Conditional Asset-Level Risk Models}

The above models focus on dynamic risk modelling of univariate return time series. In contrast, conditional asset-level risk analysis is based on a multivariate model that additionally enables to account for the dependence structure of the portfolio's assets.

\subsubsection{The RiskMetrics Approach}

The RiskMetrics (RM) model is arguable the most simple and common approach among finance practitioners for estimating time-varying covariance matrices. It utilizes an exponentially weighted moving average filter that implicitly assumes a very tight parametric specification by incorporating conditionality via the exponential smoothing of individual squared returns and cross products. The estimate for the $N \times N$ covariance matrix at time $t+1, \hat{\Sigma}_{t+1}$, is then defined by

$$
\hat{\Sigma}_{t+1}=\lambda \hat{\Sigma}_{t}+(1-\lambda) \mathbf{r}_{t} \mathbf{r}_{t}^{\prime}
$$


where $\lambda<1$ is known as the decay factor. ${ }^{15}$ The VaR and ES are then simply obtained as

$$
\begin{aligned}
\operatorname{VaR}_{t+1 \mid t}^{p} & =\left(\mathbf{w}_{t}^{\prime} \hat{\Sigma}_{t+1} \mathbf{w}_{t}\right)^{1 / 2} \Phi_{p}^{-1}, \\
\mathrm{ES}_{t+1 \mid t}^{p} & =\left(\mathbf{w}_{t}^{\prime} \hat{\Sigma}_{t+1} \mathbf{w}_{t}\right)^{1 / 2} \frac{\phi\left(\Phi_{p}^{-1}\right)}{p} .
\end{aligned}
$$

The RM model is appealing because no parameters need to be estimated, thanks to the implicit assumption of zero mean returns, a fixed smoothing parameter and conditional normality. At the same time, the RM approach is yet very restrictive, imposing the same degree of smoothness on all elements of the covariance matrix. Moreover, the RM model tends to underestimate VaR and ES under the normality assumption. We therefore employ a t-distribution instead.

\subsubsection{The Copula-GARCH Approach}

The Copula-GARCH (CG) approach proposed by Jondeau and Rockinger (2006) and Patton (2006) is based on the concept of inference from margins, i.e. dependencies between the marginal distributions are captured by a copula. In the first step, univariate $\operatorname{GARCH}(1,1)$-models are fitted to the underlying

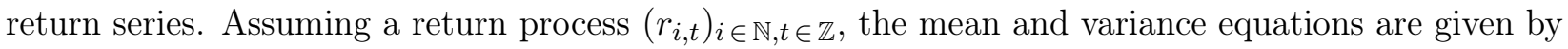

$$
\begin{aligned}
r_{i, t} & =\mu_{i}+\varepsilon_{i, t}, \\
\varepsilon_{i, t} & =z_{i, t} \sqrt{\sigma_{i, t}^{2}}, \\
z_{i, t} & \sim \mathcal{D}_{i}\left(0,1, \xi_{i}, \nu_{i}\right), \\
\sigma_{i, t}^{2} & =\omega_{i}+\alpha_{i} \varepsilon_{i, t-1}^{2}+\beta_{i} \sigma_{i, t-1}^{2},
\end{aligned}
$$

where $\omega_{i}>0, \alpha_{i} \geq 0$ and $\beta_{i} \geq 0, i=1, \ldots, N$. Moreover, $r_{i, t}$ are the returns of the $i$-th portfolio asset at time $t$, and $\mathcal{D}_{i}$ reflects the skewed-t distribution with skewness parameter $\xi_{i}$ and shape parameter $\nu_{i}$ according to Hansen (1994).

In the second step, we use a time-varying copula to estimate the marginal distributions of the asset returns together with the dependence structure. In particular, the joint distribution of the $N$ GARCH return processes can be expressed depending on an $N$-dimensional copula $C$ :

$$
F_{t}\left(\mathbf{r}_{t} \mid \boldsymbol{\mu}_{t}, \boldsymbol{\sigma}_{t}\right)=C_{t}\left(F_{1, t}\left(r_{1, t} \mid \mu_{1, t}, \sigma_{1, t}\right), \ldots, F_{N, t}\left(r_{N, t} \mid \mu_{N, t}, \sigma_{N, t}\right) \mid \mathcal{F}_{t-1}\right),
$$

where $F_{1}(\cdot), \ldots, F_{N}(\cdot)$ are the conditional marginal distributions of the return processes. The dependence structure of the margins is assumed to follow a Student-t copula with conditional correlation $\mathbf{R}_{t}$ and constant shape parameter $\eta$. We opt for the Student-t copula for modelling the dependence of financial assets since the normal copula cannot account for tail dependence. The conditional density

\footnotetext{
${ }^{15}$ In practice, $\lambda$ is typically fixed at a preset value of 0.94 when using daily returns.
} 
of the Student-t copula at time $t$ is given by:

$$
c_{t}\left(u_{i, t}, \ldots, u_{N, t} \mid \mathbf{R}_{t}, \eta\right)=\frac{f_{t}\left(F_{i, t}^{-1}\left(u_{i, t} \mid \eta\right), \ldots, F_{i, t}^{-1}\left(u_{N, t} \mid \eta\right) \mid \mathbf{R}_{t}, \eta\right)}{\prod_{i=1}^{n} f_{i}\left(F_{i, t}^{-1}\left(u_{i, t} \mid \eta\right) \mid \eta\right)},
$$

where $u_{i, t}=F_{i, t}\left(r_{i, t} \mid \mu_{i, t}, \sigma_{i, t}, \xi_{i}, \nu_{i}\right)$ is the probability integral transformation of each series by its conditional distribution $F_{i, t}$ estimated via the first stage GARCH process, $F_{i, t}^{-1}\left(u_{i, t} \mid \eta\right)$ represents the quantile transformation of the uniform margins subject to the common shape parameter of the multivariate density, $f_{t}\left(\cdot \mid \mathbf{R}_{t}, \eta\right)$ is the multivariate density of the Student-t distribution with conditional correlation $\mathbf{R}_{t}$ and shape parameter $\eta$ and $f_{i}(\cdot \mid \eta)$ is the univariate margins of the multivariate Student-t distribution with common shape parameter $\eta$. Furthermore, we allow the parameters of the conditional copula to vary through time in a manner analogous to a GARCH model for conditional variance (e.g. Patton, 2006). Specifically, we assume the dynamics of $\mathbf{R}_{t}$ to follow an Asymmetric Generalized Dynamic Conditional Correlation (AGDCC) model according to Cappiello, Engle, and Sheppard (2006).

Based on the copula estimates, we then generate $N$ sets of random pseudo-uniform variables and transform these into corresponding realizations of the error processes by using the quantile function of the margins. These simulated numbers are then used together with the conditional volatility forecast of the GARCH models to derive a Monte Carlo set of returns for each asset. By means of the portfolio's weight vector we can then compute a distribution of portfolio returns for $t+1$ which allows us to calculate VaR and ES forecasts.

The Copula-GARCH model has several advantages over more simplistic approaches. The GARCH models with skewed-t distribution applied in the first stage allow to capture the main empirical characteristics of financial asset returns. Moreover, the use of copulas in the second stage helps overcoming the deficiency of the Pearson correlation that merely captures linear relationships. In particular, copulas allow to model dependencies of portfolio assets in a more flexible way. Given the associated computational effort and complexity, however, most practitioners resort to simpler methods.

\subsection{Risk Forecast Combination}

The forecasting literature (e.g. Timmermann, 2006) generally argues that combining forecasts may enhance the predictive performance relative to standalone models because of diversification benefits, robustness against structural breaks and a reduction of the dangers of model misspecifications. While there exist various approaches to combine VaR predictions (see Bayer, 2018, for a summary), the literature is lacking a method that combines ES predictions. In this section, we propose a new technique for the combination of ES (and VaR) forecasts based on a loss function of Fissler and Ziegel (2016).

Let $\operatorname{VaR}_{m, t+1 \mid t}^{p}$ and $\mathrm{ES}_{m, t+1 \mid t}^{p}$ be the VaR and ES forecast for day $t+1$ of model $m=1, \ldots, M$

based on the information available at $t$ and $\mathbf{V a R}_{t+1 \mid t}^{p}=\left[\operatorname{VaR}_{1, t+1 \mid t}^{p}, \ldots, \operatorname{VaR}_{M, t+1 \mid t}^{p}\right]^{\prime}$ and 
$\mathbf{E S}_{t+1 \mid t}^{p}=\left[\mathrm{ES}_{1, t+1 \mid t}^{p}, \ldots, \mathrm{ES}_{M, t+1 \mid t}^{p}\right]^{\prime}$ be the vectors of all forecasts. The linear combination of the $M$ forecasts is then given by:

$$
\begin{aligned}
\operatorname{VaR}_{\text {comb }, t+1 \mid t}^{p} & =\sum_{m=1}^{M} \beta_{m, t}^{\mathrm{VaR}} \operatorname{VaR}_{m, t+1 \mid t}^{p}=\left(\mathbf{V a R}_{t+1 \mid t}^{p}\right)^{\prime} \beta_{t}^{\mathrm{VaR}} \\
\mathrm{ES}_{\mathrm{comb}, t+1 \mid t}^{p} & =\sum_{m=1}^{M} \beta_{m, t}^{\mathrm{ES}} \mathrm{ES}_{m, t+1 \mid t}^{p}=\left(\mathbf{E S}_{t+1 \mid t}^{p}\right)^{\prime} \boldsymbol{\beta}_{t}^{\mathrm{ES}}
\end{aligned}
$$

where $\beta_{t}^{\mathrm{VaR}}$ and $\boldsymbol{\beta}_{t}^{\mathrm{ES}}$ are the combination weight vectors.

\subsubsection{Simple Average}

The most naive combination approach would simply average all standalone models' forecasts. The corresponding combination weights are given by

$$
\widehat{\boldsymbol{\beta}}_{m, t}=\frac{1}{M}, \quad \forall m=1, \ldots, M .
$$

According to the mean forecasting literature (see Timmermann, 2006), this approach is empirically successful and hard to beat by more sophisticated combination methods. Therefore, we consider the simple average as a benchmark approach.

\subsubsection{FZ Loss Forecast Combination}

Given that parsimonious models often perform well in stable markets, whereas highly parameterized models show their strengths during periods of high volatility we are concerned that the simple average combination approach fails to leverage the time-dependent benefits satisfactorily. Therefore, we propose a new technique for the combination of ES (and VaR) forecasts that incorporates the most recent information into the model parameters.

In order to determine the optimal combination weights, we resort to the class of loss functions proposed by Fissler and Ziegel (2016). Similar to Patton, Ziegel, and Chen (2019), we choose the parameters of the function class in such a way that the loss differences of two forecasts are homogeneous of degree zero, given that VaR and ES are strictly negative. While Patton, Ziegel, and Chen (2019) use the FZ loss function for creating standalone risk models, we use it for the purpose of risk forecast combination. The FZ loss function is given by

$$
L_{F Z}(r, \mathrm{VaR}, \mathrm{ES}, p)=-\frac{1}{p \mathrm{ES}} \mathbb{1}(r \leq \mathrm{VaR})(\operatorname{VaR}-r)+\frac{\mathrm{VaR}}{\mathrm{ES}}+\log (-\mathrm{ES})-1 .
$$

Fissler and Ziegel (2016) show consistency of this loss function, implying that the true VaR and ES predictions minimize the expected loss. Equipped with a consistent loss function, the optimal forecast combination weights consequently minimize the expected loss of the FZ loss function,

$$
\left(\left(\beta_{t}^{\mathrm{VaR}}\right)^{*},\left(\beta_{t}^{\mathrm{ES}}\right)^{*}\right)=\underset{\beta_{t}^{V a R}, \beta_{t}^{E S}}{\arg \min } \mathbb{E}\left[L_{F Z}\left(r_{\mathrm{PF}, t+1},\left(\mathbf{V a R}_{t+1 \mid t}^{p}\right)^{\prime} \boldsymbol{\beta}_{t}^{\mathrm{VaR}},\left(\mathbf{E S}_{t+1 \mid t}^{p}\right)^{\prime} \beta_{t}^{\mathrm{ES}}\right) \mid \mathcal{F}_{t}\right]
$$


Consistent and asymptotically normal estimators of the combination weights can be obtained by minimizing the average $\mathrm{FZ}$ loss, ${ }^{16}$

$$
\left(\widehat{\boldsymbol{\beta}}_{t}^{\mathrm{VaR}}, \widehat{\boldsymbol{\beta}}_{t}^{\mathrm{ES}}\right)=\underset{\boldsymbol{\beta}_{t}^{V a R}, \boldsymbol{\beta}_{t}^{E S}}{\arg \min } \frac{1}{t} \sum_{\tau=0}^{t-1} L_{F Z}\left(r_{\mathrm{PF}, \tau+1},\left(\mathbf{V a R}_{\tau+1 \mid \tau}^{p}\right)^{\prime} \boldsymbol{\beta}_{t}^{\mathrm{VaR}},\left(\mathbf{E S}_{\tau+1 \mid \tau}^{p}\right)^{\prime} \boldsymbol{\beta}_{t}^{\mathrm{ES}}\right),
$$

which can then be used to form the combined forecast

$$
\begin{aligned}
\widehat{\operatorname{VaR}}_{\mathrm{comb}, t+1 \mid t}^{p} & =\left(\mathbf{V a R}_{t+1 \mid t}^{p}\right)^{\prime} \widehat{\boldsymbol{\beta}}_{t}^{\mathrm{VaR}}, \\
\widehat{\mathrm{ES}}_{\mathrm{comb}, t+1 \mid t}^{p} & =\left(\mathbf{E S}_{t+1 \mid t}^{p}\right)^{\prime} \widehat{\boldsymbol{\beta}}_{t}^{\mathrm{ES}} .
\end{aligned}
$$

Hence, our approach allows the combination weights to differ for VaR and ES predictions, which is favorable as the quality of a method's VaR and ES may differ. Following the forecast combination literature (Timmermann, 2006; Hansen, 2008), we impose convexity on the combination weights as this restriction typically improves upon the non-constrained estimator in terms of predictive performance. Convex weights are non-negative and sum to one, i.e. $0 \leq \beta_{m} \leq 1$ for $m=1, \ldots, M$ and $\sum_{m=1}^{M} \beta_{m}=1 .^{17}$

\section{Empirically Validating Risk Models for Tail Risk Protection}

In this section, we describe the design and the results of the empirical study to compare the various methods for portfolio risk modelling using tail risk protection strategies.

\subsection{Data and Return Synchronization}

We use a global multi-asset data set, encompassing four major market risk factors: (i) equity, (ii) fixed income, (iii) commodities and (iv) exchange rates. In particular, we utilize the following representative assets: equity futures for Nikkei 225, EURO STOXX 50, FTSE 100, SEP 500, MSCI EM, bond futures for JGB 10Y, Euro Bund, UK Gilt, US 10Y, total return indices for the commodities Oil, Gold, Copper and the spot market foreign exchange rates JPY/USD, EUR/USD, GBP/USD. The money market investment is based on the 3-month U.S. Treasury Bill. We retrieve all data from Bloomberg. All asset prices are in local currency. Portfolio returns (and associated portfolio risk figures) are computed from the perspective of a U.S. investor who is hedging any currency exposure. The sample spans from 02 January 1991 to 31 March 2017, giving rise to 6,847 daily return observations for each

\footnotetext{
${ }^{16} \mathrm{~A}$ proof of consistency and asymptotic normality of the presented estimators is similar to the one in Patton, Ziegel, and Chen (2019).

${ }^{17}$ We use an optimization procedure similar to that of Engle and Manganelli (2004). We first generate $10^{5}$ vectors of parameters from a uniform random number generator such that the convex weight restriction is fulfilled. For each of these vectors, we compute the average loss from the FZ loss function and select the 10 vectors that produce the lowest average loss as initial values for the optimization routine. Using the augmented Lagrange multiplier method with a sequential quadratic programming interior algorithm according to Ye (1987) we minimize the average loss for each of the 10 resulting vectors and select the vector producing the lowest average loss as the final parameter vector.
} 
series.

To calculate portfolio risk figures, we assume a few static strategic allocations of portfolio weights. Alternatively, we could consider a dynamic weight structure driven by a tactical asset allocation component, complicating to determine whether an increase in performance is due to superior risk forecasts or due to predictability of the tactical component. As a base case we use a broadly diversified, conservative multi-asset portfolio, but we also investigate four alternative allocations: a pure equity portfolio, a pure bond portfolio, a 30/70 equity/bond portfolio and 60/40 equity/bond portfolio. Table I reports the corresponding allocation of portfolio weights as well as the descriptive statistics of the log returns of each asset and portfolio: all time series exhibit the typical features of financial assets such as fat tails and non-normality.

[Table I about here]

When modelling risk using international daily return data, one has to properly account for different market closing times. ${ }^{18}$ Even worse, for some markets trading times do not overlap at all, as is the case for the U.S. and Japan. Obviously, these differences will make equity markets appear less (cor)related than they actually are. As a result, portfolio risk estimates will overstate the diversification benefit attached to investing across these assets (see Scholes and Williams, 1977; Lo and MacKinlay, 1990; Burns, Engle, and Mezrich, 1998; Scherer, 2013). Ideally, daily returns can be computed for all series using the same time stamp. This approach, however, is hardly feasible, even when using high-frequency data. Instead, the literature suggests synchronizing daily returns by extrapolating asset prices for those markets that close earlier, based on information from markets that close latest. While Burns, Engle, and Mezrich (1998) use a first-order vector moving average model with a multivariate GARCH covariance matrix to estimate synchronized returns, Audrino and Bühlmann (2004) employ a simple first-order vector autoregressive model (see Appendix A for details on the return synchronization methodology). We follow the latter approach due to its computational efficiency.

Based on our sample, we compare the synchronized daily returns to the original ones. Table II shows the descriptive statistics of the original and synchronized daily returns. We observe that differences in the mean are only marginal, whereas volatilities are slightly higher when synchronizing. Thus, the return characteristics of the original data are maintained.

[Table II about here]

To check the effectiveness of synchronization, we have identified the correlation matrices of both return types (not shown). For the synchronized daily returns, the chosen VAR(1) model is successfully re-correlating the within-asset class correlations. While equity correlations are no longer underestimated, equity-bond correlations tend to be more negative when using synchronized returns.

\footnotetext{
${ }^{18}$ The opening times of the markets in our sample are as follows: Japanese markets are open from 19:00(-1) to 1:00 ET, EU/UK markets from 3:00 to 11:30 ET, and U.S. markets open from 09:30 to 16:15 ET.
} 
Hence, the improved equity-bond diversification could mitigate the pick-up in equity risk. However, we learn that the latter effect dominates and unreported results evidence an increase of portfolio risk figures that average to $15 \%$ for the conservative multi-asset portfolio. ${ }^{19}$ These findings are in line with Scholes and Williams (1977) and Lo and MacKinlay (1990).

\subsection{Estimating Portfolio Risk}

The empirical study considers one day-ahead estimation of the conditional VaR and ES at a $1 \%$ confidence level, being consistent with the portfolio rebalancing frequency of the considered tail risk protection strategies. Like Kuester, Mittnik, and Paolella (2006) and Taylor (2008), we use a moving window of 1,000 observations to re-estimate parameters for the various standalone risk methods on a daily basis. Similarly, forecast combination weights are re-estimated daily using a moving window of 500 observations. Unreported results show that the combination weights are robust to the window length. Given this decision, the out-of-sample estimation period ranges from 02 October 1996 to 31 March 2017 consisting of 5,348 daily VaR and ES forecasts for each method (standalone and combination) and portfolio.

\subsubsection{Standalone Risk Forecasts}

Figure 1 presents the predicted 1\%-VaR and associated ES figures of the standalone risk models for the multi-asset portfolio over the whole out-of-sample period. The figures show that the average ES was estimated at around $-2 \%$, rising to some $-0.7 \%$ in the mid 2000 s, and attaining extreme values around $-12 \%$ during the financial crisis in late 2008. Corresponding VaR figures are higher by construction. Like volatility, ES (and VaR) fluctuate substantially over time. We further observe that simple methods like historical simulation and Cornish-Fisher approximation produce forecasts that take some time to adjust to current market conditions, whereas more flexible risk models are more sensitive and quicker to react to the prevailing risk environment.

[Figure 1 about here]

\subsubsection{Combination Weights and Combination Risk Forecasts}

To foster intuition with regard to how the FZ loss combination approach estimates the combination weights and selects the standalone models, Figure 2 shows the combination weights for ES and VaR forecasts. Panel (a) exhibits the time evolution of the out-of-sample weights for the multi-asset portfolio. ES and VaR weights are not restricted to be identical, and we indeed observe different weight patterns over the sample period. While we document an average weight overlap of $64.1 \%$ when comparing ES and VaR combination weights through time we note that we still can observe periods

\footnotetext{
${ }^{19}$ Note that the synchronized returns are used for estimating and forecasting VaR and ES, but not for out-of-sample evaluation. Instead, we use the original returns for assessing the statistical validity of the risk forecasts as well as their performance in the tail risk protection strategies.
} 
with zero overlap. Although the composition of the estimated weights is sensitive to the current market conditions for both risk measures, VaR weights are slightly less volatile than ES weights. On average, this difference translates to a daily weight change of $8.8 \%$ for the ES weights and $7.4 \%$ for the VaR weights. In terms of weight composition, we find the Copula-GARCH model to be the most important component in the FZ loss combination approach, see Panel (b) giving the average weights for all portfolios over the out-of-sample period. The average ES weight for the Copula-GARCH approach ranges from $27 \%$ for the bond portfolio to $51 \%$ for the multi-asset portfolio. Another important component is the RiskMetrics forecast, even though it does not perform well individually (cf. Section 4.3). Given an estimation error of zero, the RiskMetrics model serves as a stabilizing component. On average, the ES estimation weights for all other standalone models lie between $1 \%$ and $20 \%$. We also see that the model weights differ across portfolios, suggesting that a data-driven selection of the standalone models may offer advantages compared to a simple average forecast.

[Figure 2 about here]

In addition to the characteristics of the combination weights, Figure 2 also gives the forecasts of the FZ loss (cf. Panel (c)) and the simple average combination approach (cf. Panel (d)) over time. As expected, the FZ loss combination forecasts are slightly more sensitive to the prevailing risk environment compared to the average forecast as less weight is, on average, given to simple methods such as historical simulation and Cornish-Fisher approximation.

\subsection{Statistical Validity of Risk Forecasts}

To assess forecasting performance from an econometric perspective we perform various Value-at-Risk and Expected Shortfall tests proposed in the literature. The objective of such statistical tests is to consider the ex ante portfolio risk forecasts from a specific model and compare them with the ex post realized portfolio returns.

\subsubsection{Value-at-Risk Tests}

Testing VaR forecasts boils down to evaluating the distribution of VaR violations. That is, one needs to count and investigate those realized return observations that fall below the predicted VaR-level for a given estimation period. For instance, there should be 2.5 violations in a set of 250 forecasts of daily 1\%-VaRs per year. The test for unconditional coverage (UC) of Kupiec (1995) assesses whether the frequency of violations is consistent with the quantile of loss that the VaR measure is intended to reflect. However, this test does not account for serial independence of the number of violations. In this vein, the conditional coverage (CC) test of Christoffersen (1998) offers a remedy by jointly testing the frequency as well as the independence of violations, assuming that VaR violations are modelled with a first-order Markov chain. This test could reject a VaR model that generates too many clustered violations. As the original likelihood ratio test of Christoffersen (1998) has inferior size and power properties compared to more recent alternatives (see Berkowitz, Christoffersen, and Pelletier, 2011), we also consider the dynamic quantile (DQ) test of Engle and Manganelli (2004). 
Specifically, the authors propose a regression-based test that checks whether VaR estimates satisfy the criteria of unbiasedeness, independent violations and independence of the quantile estimates. To account for clustering of extremes we further consider the duration (DU) test of Christoffersen and Pelletier (2004), which examines the duration between violations by testing the null hypothesis that the duration between violations is exponentially distributed against a Weibull alternative. More recently, Patton, Ziegel, and Chen (2019) proposed the generalized residual (GR) test. It is based on simple regressions of standardized versions of the "generalized residuals" (as given in Equation (24)), on elements of the information set available at the time the forecast was made. As these standardized generalized residuals are conditionally mean zero under the correct specification, forecast optimality can be assessed by testing that all parameters in these regressions are zero, against a two-sided alternative.

\subsubsection{Expected Shortfall Tests}

The GR test is also applicable to test ES predictions, because the generalized residuals are derived from the FZ loss function, which incorporates both VaR and ES (cf. Equation (25)). A close cousin of the GR test is the ES regression test (ESR) of Bayer and Dimitriadis (2018) which, in contrast, only needs ES forecasts as input parameters. It is based on a regression framework modelling the conditional ES as a linear function, where returns are used as the response variable and ES forecasts as the explanatory variable including an intercept term. For correct ES forecasts, the intercept and slope parameters should be equal to zero and one, respectively. A Wald statistic is then employed to test for these parameter values.

One of the first and most frequently used ES tests is the exceedance residual (ER) test of McNeil and Frey (2000). This testing procedure is based on the ES residuals that exceed VaR, $e r_{t}=\left(r_{\mathrm{PF}, t}-\widehat{\mathrm{ES}}_{t}\right) \mathbb{1}\left(r_{\mathrm{PF}, t} \leq \widehat{\mathrm{VaR}}_{t}\right)$, which should have zero mean under the null hypothesis of a correctly specified risk model. Using a bootstrap hypothesis test it is tested whether the expected value of the exceedance residuals, $\mathbb{E}\left[e r_{t}\right]$, is zero. In addition, we consider the conditional conditional calibration (CAL) test of Nolde and Ziegel (2017) for testing ES. This approach is based on a Wald-type test statistic that uses moment functions of VaR and ES. ${ }^{20}$

\subsubsection{Empirical Evidence}

Table III presents the p-values from the above VaR and ES tests. Entries greater than 0.10 indicate no evidence against optimality at the $10 \%$ significance level. Our main findings are as follows: first, we find the simple methods including historical simulation (HS), Cornish-Fisher approximation (CFA) and RiskMetrics to struggle in most of the tests. Although the HS and the CFA methods show a conclusive number of violations over whole the sample period (close to the expected number of 53 violations) and therefore pass the unconditional coverage test, they fail the remainder of VaR tests because the violations are not occurring independently, but rather in clusters. Given that a correctly

\footnotetext{
${ }^{20}$ See Bayer and Dimitriadis (2018) for a rigorous discussion of most of the applied Expected Shortfall tests.
} 
specified VaR model is the basis of estimating ES, the subsequent ES tests may be considered useless. The RiskMetrics approach fails most tests due to the large deviation from the expected number of violations (72 realized violations).

[Table III about here]

Second, we find the more sophisticated standalone models (except for the CARE approach) to pass most of the VaR and ES tests. Notably, none of the models passes all tests at the $10 \%$ significance level. Among the combination models, we provide evidence that the simple average approach delivers decent results by passing most of the tests. The newly proposed FZ loss approach is, however, even more convincing: it is the only model clearly passing all VaR and ES tests.

In addition to the number of violations and the p-values from the various tests, we show the average out-of-sample losses, based on the FZ loss function from Equation (46) and the piecewiselinear or "tick" loss function (only appropriate for VaR forecast evaluation), see the third and fourth column of Table III. The FZ loss forecast combination approach is the preferred model, exhibiting the lowest value for both loss function. As expected, the HS and CFA models are the worst models. While average losses are useful to eyeball OOS forecast performance, we still need to investigate whether the gains are statistically significant. Table IV presents t-statistics of modified Diebold-Mariano tests on the loss differences using the FZ loss function, according to Diebold and Mariano (1995); Harvey, Leybourne, and Newbold (1997); Patton, Ziegel, and Chen (2019). ${ }^{21}$ The tests are conducted and repeated as "row model minus column model", such that a positive number indicates that the column model outperforms the row model. All entries for the FZ loss forecast combination approach are positive, seeing this model to outperform all competing models. Also, this outperformance is highly significant for all comparisons, with DM t-statistics between 2.91 and 6.65. The second and third best model according to the DM tests are the Copula-GARCH model and the EVT approach. The two are not statistically different from each other and are dominated by the FZ loss combination approach only. In a nutshell, we hence find the FZ loss forecast combination approach to dominate both the more sophisticated standalone risk models as well as the simple mean combination approach. ${ }^{22}$

[Table IV about here]

\subsection{The Economic Relevance of Risk Forecasting for Tail Risk Protection}

We consider two steps when evaluating the various risk models in the tail risk protection framework. First, we analyze the historical path of each strategy. That is, assessing how each strategy would

\footnotetext{
${ }^{21}$ The outcomes of the different VaR and ES tests represent good examples of a problem highlighted in Nolde and Ziegel (2017). All of the more sophisticated models pass most of the goodness-of-fit tests, complicating the discussion of their relative performance. The comparative Diebold-Mariano tests, are therefore an important element of the overall testing framework.

${ }^{22}$ Note that the testing results and rankings for the other portfolios are qualitatively similar to those for the multi-asset portfolio discussed here.
} 
have performed when implemented over the whole out-of-sample period. For this, we assume an investment horizon of one calendar year - a typical choice of institutional and private investors alike (see Benartzi and Thaler, 1995). For the DPPI strategy, the floor is then adjusted to the current portfolio value at the start of each year to initialize the cushion. This procedure helps to mitigate the lock-in effect.

Analyzing the historical path suffers from path dependency; therefore, we additionally conduct a historical block-bootstrap analysis ${ }^{23}$ in the second step. Following Annaert, Van Osselaer, and Verstraete (2009); Bertrand and Prigent (2011); Dichtl and Drobetz (2011); Dichtl, Drobetz, and Wambach (2017), we draw blocks of 250 subsequent daily portfolio and risk-free returns on a rolling window basis and implement the tail risk protection strategies in each draw. Thus, we obtain 5,597 overlapping yearly returns as basis for the comparison of our methods. Intuitively, this historical block-bootstrap approach enables us to assess a strategy's robustness with respect to alternative entry dates. Moreover, the available data is used in the most efficient way while preserving all dependency effects in the series, such as autocorrelation and conditional heteroskedasticity (see Dichtl and Drobetz, 2011).

As the objective of tail risk protection strategies is twofold-providing downside protection while still enjoying the upside potential of the risky portfolio - the performance should be evaluated accordingly. Alongside standard measures like the Sharpe ratio and maximum drawdown we therefore employ specific downside risk measures commonly used in the portfolio insurance literature such as the Calmar, Sortino or Omega ratios (see Bertrand and Prigent, 2011). ${ }^{24}$

We implement the tail risk protection strategies without short sales or leverage and assume round-trip transaction costs of 10 basis points. To avoid portfolio shifts triggered by rather small market movements, we also apply a trading filter of $2 \%$, acting only on exposure changes in excess of 2\% (cf. Dichtl, Drobetz, and Wambach, 2017).

\subsubsection{Tail Risk Protection via Risk Targeting}

Figure 3 illustrates the performance of the ES targeting strategy for the historical path and the historical block-bootstrap, ${ }^{25}$ based on the $1 \%$-ES of the FZ loss combination approach. The underlying is the multi-asset portfolio and we target an ES level of 1.5\%, which is a reasonable assumption given the conservative underlying. Panel (a) shows the evolution of the protected portfolio, the underlying multi-asset strategy and a money market investment over the out-of-sample period from 1994 to 2017.

\footnotetext{
${ }^{23}$ This method is sometimes referred to as historical simulation, see Dichtl and Drobetz (2011).

${ }^{24}$ While the Calmar ratio is defined as the ratio of annualized return over the absolute value of the maximum drawdown, the Sortino ratio is the difference of mean return and minimum acceptable return (here: zero) divided by downside deviation (that measures the variability of underperformance below a minimum target rate). The Omega ratio is calculated by dividing the upper partial moment of degree one by the lower partial moment of degree one. Lower (upper) partial moments indicate the return potential below (above) a predefined threshold return (here: zero). See Bertrand and Prigent (2011) for details on these performance risk measures.

${ }^{25}$ Note that volatility or VaR targeting strategies deliver similar results. To be consistent with the DPPI strategy that is based on ES we solely report the results for the ES targeting strategy.
} 
We observe a decrease in exposure of the ES targeting strategy during the financial market crisis in 2008 , thus avoiding the huge drawdowns of the underlying but also reducing upside participation at the end of the sample period.

[Figure 3 about here]

Panel (b) shows the distribution of simulated yearly returns of the protected portfolio in comparison with a pure buy-and-hold portfolio investment strategy. We see that the distribution of the ES targeting strategy is shifted to the right, thus reducing the mass in the left tail. However, this reduction comes at the cost of some return potential in the upper right tail.

Table V complements the previous chart with the estimation results of the ES targeting strategy based on all different 1\%-ES forecasts for the historical path and the historical block-bootstrap. Panel A reports the results for the historical path. We find a similar size of risk-adjusted returns (cf. Sharpe ratio), but lower maximum drawdowns and thus higher Calmar ratios for all risk methods compared to the underlying. These figures confirm the ability of the ES targeting strategy to reduce downside risk. Comparing across risk models, we observe the best risk-adjusted performance (measured in Sharpe ratio) and downside risk measures (measured in Calmar ratio) for the Copula-GARCH (CG), the hybrid GAS/GARCH (Hyb-GAS) and the FZ loss approach (FZ). Thus, our results indicate that the ES targeting strategy is more profitable when using these more flexible methods. This finding is confirmed by the historical block-bootstrap analysis shown in Panel B. We observe higher Omega ratios for CG, Hyb-GAS and FZ (5.22, 5.07 and 5.25); comparing these figures to 4.45 for the historical simulation (HS) method, for example. ${ }^{26}$ Also in terms of Sharpe and Sortino ratios, these three approaches outperform all other risk models.

\section{[Table $\mathrm{V}$ about here]}

As the results of the ES targeting strategy may be sensitive to the choice of portfolio allocation and risk target, we also investigate the strategy using different underlying portfolios - a pure equity, a pure bond, a 30/70 equity/bond and a 60/40 equity/bond portfolio in addition to the multiasset portfolio - at various ES target levels $(1 \%, 1.25 \%, 1.5 \%, 1.75 \%, 2 \%)$. Table VI reports the corresponding results. Assuming appropriate portfolio-specific ES target levels, ${ }^{27}$ most portfolios benefit from the more flexible methods showing higher Calmar ratios (historical path, cf. Panel A) and Omega ratios (historical block-bootstrap, cf. Panel B), respectively. The same holds true for the robustness checks with respect to the risk target level. For most levels and for both analyses,

\footnotetext{
${ }^{26}$ Note that we rely on the Omega ratio rather than the mean of the yearly Calmar ratios in the historical blockbootstrap analysis. While the Calmar ratio is based on daily returns (and thus needs to be transformed via the mean), the Omega ratio is usually calculated for longer-horizon returns such as yearly returns and is therefore more appropriate for the historical block-bootstrap analysis (see Bertrand and Prigent, 2011; Dichtl, Drobetz, and Wambach, 2017).

${ }^{27}$ We choose the following portfolio-specific ES target levels: $1.5 \%$ for the multi-asset portfolio, $4 \%$ for the pure equity portfolio, $1.5 \%$ for the pure bond portfolio, $1.5 \%$ for the $30 / 70$ equity/bond portfolio and $2.5 \%$ for the $60 / 40$ equity/bond portfolio.
} 
historical path and historical block-bootstrap, we find superior performance of the sophisticated models (such as FZ loss approach) in terms of Calmar and Omega ratio.

[Table VI about here]

\subsubsection{Tail Risk Protection via DPPI}

While the ES targeting strategy is able to mitigate downside risk to some extent, it falls short in clearly reducing maximum drawdown. A stricter way to limit downside risk is the DPPI strategy. Panel (a) of Figure 4 illustrates how the mechanism of a DPPI strategy generally works. The chart shows the performance of the conservative multi-asset portfolio using the DPPI strategy in relation to the floor over time. The investment exposure is mainly driven by two components: the floor and the multiplier. If the portfolio value of the underlying risky investment approaches the floor from above, i.e. the cushion shrinks, the investment exposure is reduced by shifting into the risk-free asset. Similarly, the exposure is reduced if risk estimates predict too high (overnight) risk, i.e. the multiplier decreases given that the distance to the floor is not excessive. In this example, the conditional multiplier is based on the $1 \%$-ES of the FZ loss combination approach. ${ }^{28}$

[Figure 4 about here]

Examining the whole sample period, the DPPI strategy did indeed prevent severe drawdowns. With the onset of the global financial crisis, investment exposure drops to zero, so that the portfolio value at the end of 2008 is equal to the floor. Even in the subsequent V-shaped return pattern (sudden decline followed by a rapid recovery) in early 2009 - a major impediment for portfolio insurance - the DPPI portfolio does not end up in a "cash lock". It partly participates in the subsequent recovery. On the whole, the DPPI portfolio has an average investment exposure of approximately $60 \%$ to $90 \%$, depending on the chosen risk method, and delivers slightly lower returns compared to the pure multi-asset portfolio (cf. Table VII). However, the risk-adjusted results are clearly in favor of the DPPI portfolio. This relative advantage remains when considering downside risk measures. The lower maximum drawdown of the DPPI portfolio evidences that downside protection is effectiveirrespective of the choice of the risk method. Comparing the performance of the DPPI portfolio across risk models yields less clear-cut results. Panel A of Table VII shows the corresponding results. In terms of returns we observe a 76bp difference between the best performing risk model, the Copula-GARCH model, and the weakest model, the two-factor GAS model. However, in terms of risk-adjusted returns, this spread is diminished, resulting in marginal differences across models. In particular, the Sharpe ratios range from 0.53 to 0.63 . The same conclusions can be drawn in terms

\footnotetext{
${ }^{28}$ In order to reflect the preferences of risk-averse investors, we follow Soupé, Heckel, and De Carvalho (2014) and scale the risk forecast by a term consisting of an investor's risk aversion parameter and the expected Sharpe ratio given a Constant Relative Risk Aversion (CRRA) utility function of the investor. Specifically, assuming a risk-averse investor we set the risk aversion parameter to 0.15 and the expected Sharpe ratio to 0.6.
} 
of maximum drawdown. Evaluating the risk models on the basis of the Calmar, Sortino and Omega ratios shows only marginal differences as well. The respective ranges are from 0.34 to 0.42 (Calmar), around 0.09 (Sortino), and from 1.17 to 1.19 (Omega) and suggest that even rather naive approaches did not fail to provide downside protection in the context of DPPI. This finding can be rationalized as follows: in general, few allocation changes are necessary to protect from downside risks if the DPPI strategy is reasonably calibrated. In particular, the investment exposure is reduced when approaching the floor, irrespective of the underlying risk forecast. This embedded line of defense is most likely preventing less accurate risk forecasts from impeding overall performance. As a result, any DPPI strategy dominates the underlying risky portfolio when evaluating Calmar, Sortino and Omega ratios.

[Table VII about here]

Similar to several studies (Bertrand and Prigent, 2002; Ben Ameur and Prigent, 2007; Hamidi, Jurczenko, and Maillet, 2009; Ben Ameur and Prigent, 2014; Hamidi, Maillet, and Prigent, 2014), we also benchmark the DPPI performance with multipliers based on the different risk models against the CPPI performance based on a static unconditional multiplier. In particular, the latter is calculated as the maximum loss of the underlying over the whole sample period, resulting in a multiplier of 8 . In terms of downside measures, the CPPI shows slightly better results than the competing DPPI strategies (Calmar ratio of 0.47 vs. approx. 0.41) owing to a rather defensive investment exposure (approx. 60\%). As a result, there is a severe performance drag relative to the DPPI strategies: the static multiplier underperforms in terms of mean return (4.6\% vs. approx. $6.0 \%)$. In turn, the CPPI strategy embeds a severely higher insurance premium compared to the DPPI strategy which is unfavorable from an investor's perspective.

The analysis of the historical block-bootstrap confirms these findings. Panel (b) of Figure 4 shows the distribution of the simulated yearly returns of the DPPI strategy. For comparison, we also include the return distribution of a pure buy-and-hold portfolio investment strategy. The chart clearly highlights the effect of portfolio insurance. The left tail of the return distribution is shifted towards the floor level such that downside risk is reduced - albeit at the expense of some return potential in the right tail. Panel B in Table VII reports the corresponding performance statistics. Compared to the historical path, we obtain slightly different results. Concerning the performance of the underlying, Sortino and Omega ratios increase substantially for all strategies. This finding can be explained by the fact that the massive drawdown year 2008 loses weight when performing the historical block-bootstrap. In other words, the crisis year 2008 is "averaged out" to some extent. Again, we detect only marginal differences across risk models. In essence, the results support the conclusion drawn from the historical path analysis. Dynamic proportion portfolio insurance strategies building on sophisticated risk models do a good job in protecting investors from downside risk. Given that the mechanics of the portfolio insurance strategy automatically reduce investment exposure when approaching the protection level, a less sophisticated risk forecast is mainly benefiting from this second line of defense. 
Again, we provide robustness checks with respect to the choice of portfolio allocation and floor level. We use the same portfolios as in the robustness check of the ES targeting strategy and employ the following floor levels: $93 \%, 94 \%, 95 \%, 96 \%$ and $97 \%$. The corresponding results are shown in Table VIII. Assuming appropriate portfolio-specifc floor levels ${ }^{29}$ we find no significant differences across the risk models for most portfolios when analyzing the historical path (cf. Panel A). Only for the bond and the 30/70 equity/bond portfolio we document an outperformance of the more flexible methods, such as the Copula-GARCH and the FZ loss approach, in terms of Calmar ratio. Conversely, we cannot observe a clear pattern which risk method dominates for the historical block-bootstrap analysis (cf. Panel B). Analyzing robustness with respect to the floor level delivers similar results. Notably, we observe that the less sophisticated risk models HS and CFA are superior for higher floor levels. This finding can be explained by the fact that the DPPI strategy hardly acts on the risk forecasts for tighter floor levels and thus favors more conservative methods. Overall, these robustness checks confirm our findings of the DPPI strategy for the multi-asset portfolio.

[Table VIII about here]

\section{Conclusion}

Tail risk protection strategies are an effective way to limit downside risk of a given investment portfolio while maintaing most of its upside return potential. Given the limitations of option-based hedging strategies, dynamic asset allocations strategies such as the risk targeting and the dynamic proportion portfolio insurance (DPPI) strategy are popular choices among practitioners. As the success of both dynamic strategies strongly depends on the success of forecasting (tail) risk, this paper investigates a number of forecasting models to generate portfolio risk estimates that are especially suitable in timely managing the investment exposure of these strategies. To this end, we analyze risk models both prominent in the academic literature and popular among practitioners, including simple historical simulation, the RiskMetrics approach, the Cornish-Fisher Approximation, quantile/expectile regressions, extreme value theory, the Copula-GARCH approach and dynamic GAS models. In addition to standalone models, we propose a novel ES (and VaR) forecast combination approach based on a loss function that overcomes the lack of elicitability for ES by jointly modeling ES and VaR.

Empirically, we build our analysis on a global multi-asset return data set including stocks, bonds, commodities and foreign exchange rates. To take account of different market closing times we apply a return synchronization technique by extrapolating prices of closed markets, based on information from markets which close later. It turns out that the forecasts of the proposed forecast combination approach dominates both sophisticated and more naive standalone models as well as a simple average

\footnotetext{
${ }^{29}$ Here, we choose the following portfolio-specific floor levels: $95 \%$ for the multi-asset portfolio, $80 \%$ for the pure equity portfolio, $95 \%$ for the pure bond portfolio, $95 \%$ for the $30 / 70$ equity/bond portfolio and $90 \%$ for the $60 / 40$ equity/bond portfolio.
} 
combination approach in modelling the tail of the portfolio return distribution using a comprehensive $\mathrm{VaR} / \mathrm{ES}$ testing framework. When feeding the forecasts of the different risk models into the risk targeting strategy, we show that the more flexible methods, such as the Copula-GARCH, the hybrid GAS/GARCH or the FZ loss combination approach, outperform more naive methods. For the DPPI strategy, however, we find less clear-cut results. We provide evidence that dynamic portfolio insurance strategies building on sophisticated risk models are capable of protecting investors from downside risk. However, more naive approaches are also able to provide downside protection. Given that portfolio insurance only leads to few allocation changes, simple risk models might have simply been lucky. Going forward, the more accurate FZ loss forecast combination approach appears to be more likely to help mitigate the next downturn. 


\section{References}

Acerbi, C., AND B. Szekely (2014): "Backtesting expected shortfall," Risk, 27(11), 76-81.

Acerbi, C., AND D. Tasche (2002): "On the coherence of expected shortfall," Journal of Banking E6 Finance, 26(7), 1487-1503.

Andersen, T. G., T. Bollerslev, P. F. Christoffersen, And F. X. Diebold (2006): "Volatility and correlation forecasting," in Handbook of Economic Forecasting, ed. by G. Elliott, C. W. Granger, and A. Timmermann, vol. 1, chap. 15, pp. 777-878. Elsevier.

(2013): "Financial risk measurement for financial risk management," in Handbook of the Economics of Finance, ed. by G. M. Constantinides, M. Harris, and R. M. Stulz, vol. 2, chap. 17, pp. 1127-1220. Elsevier.

Annaert, J., S. Van Osselaer, And B. Verstraete (2009): "Performance evaluation of portfolio insurance strategies using stochastic dominance criteria," Journal of Banking \& Finance, 33(2), 272-280.

Ardia, D., K. Boudt, And M. Wauters (2016): "Smart beta and CPPI performance," Finance, 37(3), 31-65.

Artzner, P., F. Delbaen, J.-M. Eber, And D. Heath (1999): "Coherent measures of risk," Mathematical Finance, $9(3), 203-228$.

Audrino, F., AND P. Bühlmann (2004): "Synchronizing multivariate financial time series," Journal of Risk, 6(2), 81-106.

Balder, S., M. Brandl, AND A. Mahayni (2009): "Effectiveness of CPPI strategies under discrete-time trading," Journal of Economic Dynamics and Control, 33(1), 204-220.

BASAK, S. (2002): "A comparative study of portfolio insurance," Journal of Economic Dynamics and Control, 26(7), 1217-1241.

Bates, J. M., AND C. W. Granger (1969): "The combination of forecasts," Journal of the Operational Research Society, 20(4), 451-468.

BAYER, S. (2018): "Combining Value-at-Risk forecasts using penalized quantile regressions," Econometrics and Statistics, 8, 56-77.

Bayer, S., And T. Dimitriadis (2018): "Regression based expected shortfall backtesting," Working Paper.

Ben Ameur, H., And J.-L. Prigent (2007): "Portfolio insurance: Determination of a dynamic CPPI multiple as function of state variables," Working paper, THEMA (University of Cergy) and ISC (Paris).

(2014): "Portfolio insurance: Gap risk under conditional multiples," European Journal of Operational Research, 236(1), 238-253.

Benartzi, S., And R. H. Thaler (1995): "Myopic loss aversion and the equity premium puzzle," Quarterly Journal of Economics, 110(1), 73-92.

Benninga, S. (1990): "Comparing portfolio insurance strategies," Financial Markets and Portfolio Management, 4(1), 20-30.

Berkowitz, J., P. Christoffersen, And D. Pelletier (2011): "Evaluating value-at-risk models with desk-level data," Management Science, 57(12), 2213-2227.

Bertrand, P., And J.-L. Prigent (2002): "Portfolio insurance: The extreme value approach to the CPPI method," Finance, 23(2), 69-86.

$1811-1823$.

Black, F., AND R. W. Jones (1987): "Simplifying portfolio insurance," Journal of Portfolio Management, 14(1), $48-51$. 33-37.

Black, F., AND A. Perold (1992): "Theory of constant proportion portfolio insurance," Journal of Economic Dynamics and Control, 16(3-4), 403-426.

Bollerslev, T. (1986): "Generalized autoregressive conditional heteroskedasticity," Journal of Econometrics, 31(3), 
$307-327$.

Bollerslev, T., B. Hood, J. Huss, And L. H. Pedersen (2018): "Risk everywhere: Modeling and managing volatility," Review of Financial Studies, 31(7), 2729-2773.

Boudt, K., B. Peterson, And C. Croux (2008): "Estimation and decomposition of downside risk for portfolios with non-normal returns," Journal of Risk, 11(2), 79-103.

Burns, P., R. F. Engle, And J. J. Mezrich (1998): "Correlations and volatilities of asynchronous data," Journal of Derivatives, 5(4), 7-18.

Cappiello, L., R. F. Engle, And K. Sheppard (2006): "Asymmetric dynamics in the correlations of global equity and bond returns," Journal of Financial Econometrics, 4(4), 537-572.

Chen, J.-S., C.-L. Chang, J.-L. Hou, And Y.-T. Lin (2008): "Dynamic proportion portfolio insurance using genetic programming with principal component analysis," Expert Systems with Applications, 35(1), $273-278$.

Christoffersen, P., AND D. Pelletier (2004): "Backtesting value-at-risk: A duration-based approach," Journal of Financial Econometrics, 2(1), 84-108.

Christoffersen, P. F. (1998): "Evaluating interval forecasts," International Economic Review, 39(4), 841-862.

Cont, R., AND P. TANkov (2009): "Constant proportion portfolio insurance in the presence of jumps in asset prices," Mathematical Finance, 19(3), 379-401.

Cooper, T. (2010): "Alpha generation and risk smoothing using managed volatility," Working Paper.

Cornish, E. A., AND R. A. Fisher (1938): "Moments and cumulants in the specification of distributions," Revue de l'Institut International de Statistique, 5(4), 307-320.

Creal, D., S. J. Koopman, And A. Lucas (2013): "Generalized autoregressive score models with applications," Journal of Applied Econometrics, 28(5), 777-795.

Dichte, H., AND W. Drobetz (2011): "Portfolio insurance and prospect theory investors: Popularity and optimal design of capital protected financial products," Journal of Banking \& Finance, 35(7), 1683-1697.

Dichtl, H., W. Drobetz, AND M. WAmbach (2017): "A bootstrap-based comparison of portfolio insurance strategies," European Journal of Finance, 23(1), 31-59.

Diebold, F. X., AND R. S. Mariano (1995): "Comparing predictive accuracy," Journal of Business ES Economic Statistics, 13(3), 253-263.

Embrechts, P., AND M. Hofert (2014): "Statistics and quantitative risk management for banking and insurance," Annual Review of Statistics and Its Application, 1, 493-514.

Emmer, S., M. Kratz, And D. TAsche (2015): "What is the best risk measure in practice? A comparison of standard measures," Journal of Risk, 18(2), 31-60.

ENGLE, R. F. (1982): "Autoregressive conditional heteroscedasticity with estimates of the variance of United Kingdom inflation," Econometrica, 50(4), 987-1007.

Engle, R. F., And S. Manganelli (2004): "CAViaR: Conditional autoregressive value at risk by regression quantiles," Journal of Business 85 Economic Statistics, 22(4), 367-381.

Fissler, T., AND J. F. Ziegel (2016): "Higher order elicitability and Osband's principle," Annals of Statistics, 44(4), 1680-1707.

Giese, G. (2012): "Optimal design of volatility-driven algo-alpha trading strategies,” Risk, 25(6), 68-73.

Gneiting, T. (2011): "Making and evaluating point forecasts," Journal of the American Statistical Association, 106(494), 746-762.

Halbleib, R., AND W. Pohlmeier (2012): "Improving the value at risk forecasts: Theory and evidence from the financial crisis," Journal of Economic Dynamics and Control, 36(8), 1212-1228.

Hallerbach, W. G. (2012): "A proof of the optimality of volatility weighting over time," Journal of Investment Strategies, 1(4), 87-99.

(2015): "Advances in portfolio risk control," in Risk-Based and Factor Investing, ed. by E. Jurczenko, vol. 1, chap. 1, pp. 1-30. Elsevier. 
Hamidi, B., C. Hurlin, P. Kouontchou, And B. Maillet (2015): “A DARE for VaR,” Finance, 36(1), 7-38.

Hamidi, B., E. Jurczenko, And B. Maillet (2009): "A CAViaR modelling for a simple time-varying proportion portfolio insurance strategy," Bankers, Markets $\&$ Investors, 102, 4-21.

Hamidi, B., B. Maillet, And J.-L. Prigent (2014): "A dynamic autoregressive expectile for time-invariant portfolio protection strategies," Journal of Economic Dynamics and Control, 46, 1-29.

Hamidi, B., B. B. Maillet, And J.-L. Prigent (2009): "A risk management approach for portfolio insurance strategies," in Proceedings of the 1st EIF International Financial Research Forum, Economica.

Hansen, B. E. (1994): "Autoregressive conditional density estimation," International Economic Review, 35, 705-730. (2008): "Least-squares forecast averaging," Journal of Econometrics, 146(2), 342-350.

Harvey, A. C. (2013): "Dynamic models for volatility and heavy tails: with applications to financial and economic time series," in Econometric Society Monographs, vol. 52. Cambridge University Press.

Harvey, D., S. Leybourne, And P. Newbold (1997): "Testing the equality of prediction mean squared errors," International Journal of Forecasting, 13(2), 281-291.

Hocquard, A., S. NG, AND N. PApageorgiou (2013): "A constant-volatility framework for managing tail risk," Journal of Portfolio Management, 39(2), 28-40.

Ilmanen, A., AND J. Kizer (2012): "The death of diversification has been greatly exaggerated," Journal of Portfolio Management, 38(3), 15-27.

JiAnG, C., Y. MA, AND Y. An (2009): "The effectiveness of the VaR-based portfolio insurance strategy: An empirical analysis," International Review of Financial Analysis, 18(4), 185-197.

Jondeau, E., And M. Rockinger (2006): "The Copula-GARCH model of conditional dependencies: An international stock market application," Journal of International Money and Finance, 25(5), 827-853.

Kirby, C., AND B. OstdieK (2012): "It's all in the timing: Simple active portfolio strategies that outperform naive diversification," Journal of Financial and Quantitative Analysis, 47(2), 437-467.

Koenker, R., And G. Bassett (1978): "Regression quantiles," Econometrica, 46(1), 33-50.

Kuester, K., S. Mittnik, And M. S. Paolella (2006): "Value-at-risk prediction: A comparison of alternative strategies," Journal of Financial Econometrics, 4(1), 53-89.

Kupiec, P. H. (1995): "Techniques for verifying the accuracy of risk measurement models," Journal of Derivatives, $3(2), 73-84$.

Lo, A. W., AND A. C. MacKinlay (1990): "An econometric analysis of nonsynchronous trading," Journal of Econometrics, 45(1-2), 181-211.

Longin, F., AND B. Solnik (1995): "Is the correlation in international equity returns constant: 1960-1990?," Journal of International Money and Finance, 14(1), 3-26.

Martin, R. D., AND R. Arora (2017): "Inefficiency and bias of modified value-at-risk and expected shortfall," Journal of Risk, 19(6), 59-84.

MCNeIL, A. J., AND R. Frey (2000): "Estimation of tail-related risk measures for heteroscedastic financial time series: An extreme value approach," Journal of Empirical Finance, 7(3), 271-300.

Nadarajah, S., B. Zhang, And S. Chan (2014): "Estimation methods for expected shortfall," Quantitative Finance, $14(2), 271-291$.

Newey, W. K., AND J. L. Powell (1987): "Asymmetric least squares estimation and testing," Econometrica, 55, 819-847.

Nolde, N., AND J. F. Ziegel (2017): "Elicitability and backtesting: Perspectives for banking regulation," Annals of Applied Statistics, 11(4), 1833-1874.

Patton, A. J. (2006): "Modelling asymmetric exchange rate dependence," International Economic Review, 47(2), $527-556$.

Patton, A. J., J. F. Ziegel, And R. Chen (2019): "Dynamic semiparametric models for expected shortfall (and value-at-risk)," Journal of Econometrics, 211(2), 388-413. 
Perchet, R., R. L. De Carvalho, T. Heckel, And P. Moulin (2015): "Predicting the success of volatility targeting strategies: Application to equities and other asset classes," Journal of Alternative Investments, 18(3), $21-38$.

Perold, A. (1986): "Constant proportion portfolio insurance," Harvard Business School.

Perold, A. F., AND W. F. Sharpe (1988): "Dynamic strategies for asset allocation," Financial Analysts Journal, $44(1), 16-27$.

Pritsker, M. (2006): "The hidden dangers of historical simulation," Journal of Banking E Finance, 30(2), 561-582.

Righi, M. B., AND P. S. Ceretta (2015): "A comparison of expected shortfall estimation models," Journal of Economics and Business, 78, 14-47.

Santos, A. A., F. J. Nogales, And E. Ruiz (2012): "Comparing univariate and multivariate models to forecast portfolio value-at-risk," Journal of Financial Econometrics, 11(2), 400-441.

Scherer, B. (2013): "Synchronize your data or get out of step with your risks," Journal of Derivatives, 20(3), 75-84.

Scholes, M., AND J. Williams (1977): "Estimating betas from nonsynchronous data," Journal of Financial Economics, 5(3), 309-327.

Shan, K., AND Y. YAng (2009): "Combining regression quantile estimators," Statistica Sinica, 19(3), $1171-1191$.

Soupé, F., T. Heckel, AND R. L. De Carvalho (2014): "Portfolio insurance with adaptive protection (PIWAP)," Journal of Investment Strategies, 5(3), 1-15.

TAYloR, J. W. (2008): "Estimating value at risk and expected shortfall using expectiles," Journal of Financial Econometrics, 6(2), 231-252.

(2019a): "Forecast combinations for value at risk and expected shortfall," International Journal of Forecasting, forthcoming.

(2019b): "Forecasting value at risk and expected shortfall using a semiparametric approach based on the asymmetric Laplace distribution," Journal of Business \& Economic Statistics, 37(1), 121-133.

Taylor, S. J. (1986): Modelling Financial Time Series. Chichester: Wiley.

Timmermann, A. (2006): "Forecast combinations," in Handbook of Economic Forecasting, ed. by G. Elliott, C. Granger, and A. Timmermann, vol. 1, chap. 4, pp. 135-196. Elsevier.

Yamai, Y., T. Yoshiba, et AL. (2002): "On the validity of value-at-risk: Comparative analyses with expected shortfall," Monetary and Economic Studies, 20(1), 57-85.

YE, Y. (1987): "Interior algorithms for linear, quadratic, and linearly constrained non-linear programming," Ph.D. thesis, Department of ESS, Stanford University.

Zangari, P. (1996): "A VaR methodology for portfolios that include options," RiskMetrics Monitor, 1, 4-12. 


\section{Appendix A Return Synchronization}

In this section, we describe the return synchronization methodology that we apply to the global multi-asset data set (see Audrino and Bühlmann, 2004). Let $S_{t_{i}, i}$ denote the continuous time price of asset $i(i=1, \ldots, N)$, where time $t_{i}$ is the closing time of market $i$ measured in local time of the base market, i.e. the market with which to synchronize. The corresponding synchronized price $S_{t, i}^{s}$ is then defined as

$$
\log \left(S_{t, i}^{s}\right)=\mathbb{S}\left[\log \left(S_{t, i}\right) \mid \mathcal{F}_{t}\right]=\mathbb{E}\left[\log \left(S_{t_{i}+1, i}\right) \mid \mathcal{F}_{t}\right], \quad t_{i} \leq t \leq t_{i}+1(t \in \mathbb{N}),
$$

where $t=t_{1}$ and $\mathcal{F}_{t}$ is the complete information of all recorded prices up to time $t$. The logarithms are used to be consistent with continuously compounded returns. Clearly, if the closing price $S$ is observed at time $t \in \mathbb{N}$, its conditional expectation given $\mathcal{F}_{t}$ is the observed price. This is the case for the assets from the base market. If the market closes before $t$, its past prices and all the other markets may be useful in predicting $S$ at time $t$. As a simplifying approximation, the authors therefore assume that, given the information $\mathcal{F}_{t}$, the best predicted log-prices at $t$ and at the nearest succeeding closing time $t_{i}+1$ remain the same, saying that future changes up to $t_{i}+1$ are unpredictable.

Then, we denote $r_{t}$ as the vector of log-returns in different markets using the multi index $t=\left(t_{1}, t_{2}, \cdots, t_{N}\right)$ and define the synchronized returns $r_{t}^{s}$ as the change in the logarithms of the synchronized prices:

$$
r_{t}=\left[\begin{array}{c}
\log \left(\frac{S_{t_{1}, 1}}{S_{t_{1}-1,1}}\right) \\
\vdots \\
\log \left(\frac{S_{t_{N}, N}}{S_{t_{N}-1, N}}\right)
\end{array}\right], \quad r_{t}^{s}=\left[\begin{array}{c}
\log \left(\frac{S_{t, 1}^{s}}{S_{t-1,1}^{s}}\right) \\
\vdots \\
\log \left(\frac{S_{t, N}^{s}}{S_{t-1, N}^{s}}\right)
\end{array}\right] .
$$

In order to estimate the relationship between the individual asset markets, the authors employ a simple "auxiliary" VAR(1) model:

$$
r_{t}=\mathbf{A} r_{t-1}+\varepsilon_{t}
$$

where the innovations $\varepsilon_{t}$ are i.i.d. $\sim \mathcal{N}(\mathbf{0}, \Sigma)$, independent from $\left\{r_{s} ; s<t\right\}$, and $\mathbf{A}$ is the matrix of VAR coefficients. We can then derive the synchronized returns as follows

$$
\begin{aligned}
r_{t}^{s} & =\log \left(\mathbf{S}_{t}^{s}\right)-\log \left(\mathbf{S}_{t-1}^{s}\right) \\
& =\mathbb{E}\left[\log \left(\mathbf{S}_{t+1}\right) \mid \mathcal{F}_{t}\right]-\mathbb{E}\left[\log \left(\mathbf{S}_{t}\right) \mid \mathcal{F}_{t-1}\right] \\
& =\mathbb{E}\left[\log \left(\mathbf{S}_{t+1}\right)-\log \left(\mathbf{S}_{t}\right) \mid \mathcal{F}_{t}\right]-\mathbb{E}\left[\log \left(\mathbf{S}_{t}\right)-\log \left(\mathbf{S}_{t-1}\right) \mid \mathcal{F}_{t-1}\right]+\log \left(\frac{\mathbf{S}_{t}}{\mathbf{S}_{t-1}}\right) \\
& =\mathbb{E}\left[r_{t+1} \mid \mathcal{F}_{t}\right]-\mathbb{E}\left[r_{t} \mid \mathcal{F}_{t-1}\right]+r_{t} \\
& =\mathbf{A} r_{t}-\mathbf{A} r_{t-1}+r_{t} \\
& =r_{t}+\mathbf{A}\left(r_{t}-r_{t-1}\right) .
\end{aligned}
$$


That is, any synchronized return $r_{t}^{s}$ is still anchored in the actual realized return $r_{t}$ plus an anticipated innovation according to the estimated VAR-relation as captured in matrix $\mathbf{A}$. The "missing" dynamics of markets closing early in the day are thus proxied according to the short-term relationship with respect to those markets closing later that day.

Sorting markets according to their closing times enables to readily formulate a restriction matrix for the VAR model such that markets are explained only by those markets with a later closing time. Given that U.S. markets are the last to close in our sample, we anchor our synchronization of daily returns in U.S. markets. Thus, the U.S. time series remain unchanged but are still included in the VAR model to serve as explanatory variables, i.e. the final set of synchronized daily returns does not build on forecasted time series for the U.S. but uses their original daily returns. Non-U.S. data is forecasted to the closing time of the U.S. market by the $\operatorname{VAR}(1)$. 
Table I

\section{Descriptive Statistics and Test Portfolio Allocations}

This table reports the descriptive statistics of the daily log-returns of the individual assets and test portfolios over the period 2/1/1991 to 31/3/2017 (including 6,847 observations). The following statistics are reported: mean, median (Med), minimum (Min), maximum (Max), standard deviation (Sd), skewness (Skew) and kurtosis (Kurt). All statistics are given in percentage, except skewness and kurtosis. In addition, we provide the static weights of the test portfolio allocations (Multi-Asset (MA), Equity (EQ), Bond (BO), 30/70 equity/bond (30/70), 60/40 equity/bond (60/40)) in percentage in the last five columns.

\begin{tabular}{|c|c|c|c|c|c|c|c|c|c|c|c|c|}
\hline & \multirow[b]{2}{*}{ Mean } & \multirow[b]{2}{*}{ Med } & \multirow[b]{2}{*}{ Min } & \multirow[b]{2}{*}{ Max } & \multirow[b]{2}{*}{$\mathrm{Sd}$} & \multirow[b]{2}{*}{ Skew } & \multirow[b]{2}{*}{ Kurt } & \multicolumn{5}{|c|}{ Portfolio weights } \\
\hline & & & & & & & & MA & EQ & $\mathrm{BO}$ & $30 / 70$ & $60 / 40$ \\
\hline \multicolumn{13}{|c|}{ Individual assets } \\
\hline \multicolumn{13}{|l|}{ Stocks } \\
\hline Nikkei 225 & -0.00 & 0.00 & -14.0 & 18.82 & 1.51 & -0.20 & 119.32 & 5 & 9.8 & 0 & 2.9 & 5.9 \\
\hline Euro STOXX 50 & 0.03 & 0.05 & -9.44 & 11.38 & 1.39 & -0.12 & 88.46 & 5 & 12.5 & 0 & 3.8 & 7.5 \\
\hline MSCI EM & 0.02 & 0.08 & -9.99 & 10.07 & 1.14 & -0.52 & 108.25 & 5 & 15.2 & 0 & 4.6 & 9.1 \\
\hline FTSE 100 & 0.02 & 0.00 & -9.70 & 9.58 & 1.13 & -0.15 & 86.28 & 5 & 9.9 & 0 & 3.0 & 5.9 \\
\hline $\mathrm{S} \& \mathrm{P} 500$ & 0.02 & 0.03 & -10.4 & 13.20 & 1.13 & -0.15 & 142.71 & 15 & 52.6 & 0 & 15.8 & 31.6 \\
\hline \multicolumn{13}{|l|}{ Bonds } \\
\hline JGB $10 \mathrm{Y}$ & 0.01 & 0.00 & -1.55 & 2.18 & 0.25 & -0.28 & 82.85 & 10 & 0 & 10 & 7 & 4 \\
\hline Euro Bund & 0.02 & 0.01 & -1.73 & 1.96 & 0.33 & -0.19 & 48.7 & 10 & 0 & 20 & 14 & 8 \\
\hline UK Gilt & 0.01 & 0.00 & -2.34 & 3.65 & 0.41 & 0.06 & 62.65 & 10 & 0 & 10 & 7 & 4 \\
\hline US $10 \mathrm{Y}$ & 0.01 & 0.00 & -2.63 & 3.53 & 0.37 & -0.10 & 62.63 & 10 & 0 & 40 & 28 & 16 \\
\hline \multicolumn{13}{|l|}{ Commodities } \\
\hline Oil & 0.00 & 0.00 & -38.4 & 13.34 & 2.16 & -0.95 & 206.1 & 5 & 0 & 0 & 0 & 0 \\
\hline Gold & 0.02 & 0.00 & -9.81 & 8.84 & 1.01 & -0.17 & 112.25 & 5 & 0 & 0 & 0 & 0 \\
\hline Copper & 0.02 & 0.00 & -11.7 & 11.65 & 1.61 & -0.19 & 76.55 & 5 & 0 & 0 & 0 & 0 \\
\hline \multicolumn{13}{|c|}{ Foreign exchange rates } \\
\hline EUR/USD & -0.00 & 0.00 & -3.38 & 3.93 & 0.62 & 0.04 & 52.18 & 15 & 0 & 20 & 14 & 8 \\
\hline GBP/USD & -0.01 & 0.00 & -7.94 & 5.24 & 0.60 & -0.49 & 113.7 & 15 & 0 & 10 & 7 & 4 \\
\hline JPY/USD & 0.00 & 0.00 & -4.07 & 7.06 & 0.68 & 0.46 & 83.9 & 15 & 0 & 10 & 7 & 4 \\
\hline \multicolumn{13}{|l|}{ Asset portfolios } \\
\hline Multi-asset & 0.02 & 0.03 & -3.83 & 3.67 & 0.46 & -0.27 & 93.07 & - & - & - & - & - \\
\hline Equity & 0.02 & 0.06 & -8.42 & 10.24 & 0.93 & -0.32 & 130.9 & - & - & - & - & - \\
\hline Bond & 0.02 & 0.02 & -1.93 & 1.98 & 0.36 & -0.12 & 47.99 & - & - & - & - & - \\
\hline $30 / 70$ & 0.02 & 0.03 & -2.72 & 2.76 & 0.34 & -0.15 & 79.18 & - & - & - & - & - \\
\hline $60 / 40$ & 0.02 & 0.04 & -5.12 & 6.04 & 0.55 & -0.24 & 124.77 & - & - & - & - & - \\
\hline 3-M US T-Bill & 0.01 & 0.01 & -0.00 & 0.02 & 0.01 & 0.06 & 13.86 & - & - & - & - & - \\
\hline
\end{tabular}




\section{Table II}

\section{Synchronized vs. Original Daily Returns}

This table reports descriptive statistics for the synchronized and original daily returns. As we anchor the synchronization of daily returns in U.S. markets, the U.S. time series remain unchanged and are thus not reported. Non-U.S. data is forecasted to the closing time of the U.S. market by the VAR(1). All figures are given in percentage.

\begin{tabular}{|c|c|c|c|c|c|c|}
\hline & Nikkei 225 & JGB10Y & Euro Bund & UK Gilt & $\begin{array}{r}\text { EURO } \\
\text { STOXX } 50\end{array}$ & FTSE 100 \\
\hline \multicolumn{7}{|l|}{ Original returns } \\
\hline Mean & -0.0035 & 0.0136 & 0.0165 & 0.0149 & 0.0305 & 0.0178 \\
\hline Standard deviation & 1.5109 & 0.2486 & 0.3341 & 0.4098 & 1.3875 & 1.1306 \\
\hline First-order autocorrelation & -5.3639 & -3.3298 & 0.2096 & 0.9714 & -2.6876 & -2.1746 \\
\hline \multicolumn{7}{|l|}{ Synchronized returns } \\
\hline Mean & -0.0034 & 0.0136 & 0.0165 & 0.0149 & 0.0307 & 0.0179 \\
\hline Standard deviation & 1.6338 & 0.2561 & 0.3581 & 0.4386 & 1.5829 & 1.2916 \\
\hline First-order autocorrelation & -13.7891 & -6.314 & -6.3808 & -5.5118 & -12.9119 & -12.8367 \\
\hline
\end{tabular}


Table III

VaR and ES Backtesting

This table shows the results of VaR and ES tests for evaluating 1\%-VaR and 1\%-ES predictions based on the eleven forecasting models applied to the multi-asset portfolio over the out-of-sample period from 02/10/1996 to 31/3/2017. For testing VaR we include the unconditional coverage (UC), the conditional coverage (CC), the dynamic quantile (DQ) and the duration (DU) test. For testing ES we resort to the exceedance residual (ER), the conditional calibration $(\mathrm{CAL})$ and the ES regression (ESR) test. The generalized residual (GR) test allows to test both VaR and ES. We report p-values that are in bold if greater than 0.10 , indicating no evidence against optimality at the $10 \%$ significance level. Values between 0.05 and 0.10 are in italics. We further report the number of realized VaR violations (second column) and the average loss using the FZ loss function (third column) and the tick loss function (fourth column), the latter two being scaled by 100. The lowest average loss in each column is highlighted in bold, the second-lowest in italics. The expected number of violations is 53 over the whole out-of-sample period.

\begin{tabular}{|c|c|c|c|c|c|c|c|c|c|c|c|c|}
\hline & \multirow[b]{2}{*}{ Viol } & \multirow[b]{2}{*}{ FZ } & \multirow[b]{2}{*}{ Tick } & \multicolumn{5}{|c|}{ VaR tests } & \multicolumn{4}{|c|}{ ES tests } \\
\hline & & & & $\mathrm{UC}$ & $\mathrm{CC}$ & DQ & DU & GR & ER & CAL & ESR & GR \\
\hline \multicolumn{13}{|l|}{ Standalone models } \\
\hline Historical Simulation & 57 & 60.11 & 1.91 & 0.63 & 0.00 & 0.00 & 0.00 & 0.00 & 0.40 & 0.44 & 0.10 & 0.02 \\
\hline Cornish-Fisher-Approximation & 44 & 58.15 & 1.87 & 0.18 & 0.00 & 0.00 & 0.00 & 0.00 & 0.07 & 0.01 & 0.21 & 0.00 \\
\hline RiskMetrics & 72 & 38.84 & 1.51 & 0.02 & 0.01 & 0.00 & 0.83 & 0.01 & 0.12 & 0.00 & 0.01 & 0.01 \\
\hline CARE & 58 & 46.67 & 1.58 & 0.54 & 0.75 & 0.81 & 0.03 & 0.00 & 0.03 & 0.67 & 0.02 & 0.00 \\
\hline Extreme Value Theory & 44 & 36.25 & 1.51 & 0.18 & 0.28 & 0.08 & 0.24 & 0.27 & 0.46 & 0.35 & 0.49 & 0.25 \\
\hline Copula-GARCH & 71 & 34.79 & 1.48 & 0.02 & 0.05 & 0.00 & 0.15 & 0.16 & 0.38 & 0.11 & 0.13 & 0.14 \\
\hline One-Factor-GAS & 54 & 41.83 & 1.57 & 0.94 & 0.57 & 0.03 & 0.30 & 0.00 & 0.88 & 0.99 & 0.29 & 0.00 \\
\hline Two-Factor-GAS & 64 & 46.66 & 1.62 & 0.16 & 0.05 & 0.00 & 0.02 & 0.25 & 0.45 & 0.28 & 0.06 & 0.18 \\
\hline Hybrid-GAS/GARCH & 55 & 40.90 & 1.55 & 0.83 & 0.85 & 0.40 & 0.79 & 0.03 & 0.36 & 0.93 & 0.15 & 0.10 \\
\hline \multicolumn{13}{|l|}{ Combination models } \\
\hline Average & 49 & 38.96 & 1.54 & 0.53 & 0.64 & 0.00 & 0.01 & 0.10 & 0.89 & 0.68 & 0.32 & 0.13 \\
\hline FZ loss & 56 & 28.11 & 1.41 & 0.73 & 0.83 & 0.42 & 0.83 & 0.94 & 0.83 & 0.80 & 0.90 & 0.98 \\
\hline
\end{tabular}


Table IV

Diebold-Mariano Tests

This table presents t-statistics from modified Diebold-Mariano tests according to Harvey, Leybourne, and Newbold (1997) comparing the average losses, using the FZ loss function, over the out-of-sample period from 02/10/1996 to $31 / 3 / 2017$, for the eleven risk models, based on the multi-asset portfolio. The first nine rows correspond to the standalone models: historical simulation (HS), RiskMetrics (RM), Cornish-Fisher approximation (CFA), conditional autoregressive expectile model (CARE), extreme value theory (EVT), Copula-GARCH (CG), one-factor GAS (1FGAS), two-factor GAS (2F-GAS) and hybrid-GAS/GARCH (Hyb-GAS) model. The last two rows correspond to the combination models: the simple average forecast (Average) and the proposed FZ loss approach (FZ). A positive value indicates that the row model's average loss is higher than that for the column model. Values greater than 1.96 in absolute value indicate that the average loss difference is significantly different from zero at the $95 \%$ confidence level. Values along the main diagonal are all identically zero and are omitted for interpretability.

\begin{tabular}{lccccccrrrrrr}
\hline & HS & CFA & RM & CARE & EVT & CG & 1F-GAS & 2F-GAS & Hyb-GAS & Average & FZ \\
\hline HS & & 0.33 & 1.91 & 1.32 & 2.19 & 2.26 & 1.83 & 1.37 & 1.98 & 2.12 & 2.91 \\
CFA & -0.33 & & 2.94 & 1.97 & 3.62 & 3.69 & 3.21 & 2.14 & 3.47 & 3.95 & 5.01 \\
RM & -1.91 & -2.94 & & -1.72 & 1.25 & 1.40 & -0.95 & -2.00 & -0.69 & -0.04 & 3.74 \\
CARE & -1.32 & -1.97 & 1.72 & & 2.64 & 2.95 & 1.39 & 0.00 & 1.68 & 2.32 & 5.08 \\
EVT & -2.19 & -3.62 & -1.25 & -2.64 & & 0.61 & -2.37 & -3.00 & -1.93 & -1.49 & 5.09 \\
CG & -2.26 & -3.69 & -1.40 & -2.95 & -0.61 & & -2.65 & -3.44 & -2.34 & -1.70 & 3.20 \\
1F-GAS & -1.83 & -3.21 & 0.95 & -1.39 & 2.37 & 2.65 & & -1.86 & 0.63 & 1.94 & 6.65 \\
2F-GAS & -1.37 & -2.14 & 2.00 & 0.00 & 3.00 & 3.44 & 1.86 & & 2.48 & 3.14 & 5.84 \\
Hyb-GAS & -1.98 & -3.47 & 0.69 & -1.68 & 1.93 & 2.34 & -0.63 & -2.48 & & 1.19 & 5.51 \\
\hline Average & -2.12 & -3.95 & 0.04 & -2.32 & 1.49 & 1.70 & -1.94 & -3.14 & -1.19 & & 6.48 \\
FZ & -2.91 & -5.01 & -3.74 & -5.08 & -5.09 & -3.20 & -6.65 & -5.84 & -5.51 & -6.48 & \\
\hline
\end{tabular}




\section{Table V}

\section{Risk Targeting for Multi-Asset Portfolio}

This table shows the backtesting results of the risk targeting strategy based on different 1\%-ES forecasts for the historical path (Panel A) and the historical block-bootstrap (Panel B) over the sample period 1996 to 2017. For comparison, we include the performance of the underlying multi-asset portfolio and the money market investment. We target an ES of 1.5\% over the whole out-of-sample period. We report the annualized mean return (Return), annualized standard deviation (Sd), Sharpe ratio (SR), maximum drawdown (MDD), Calmar ratio, Sortino ratio, Omega ratio, participation in the risky multi-asset portfolio (Part), turnover (TO) and the 1\% Expected Shortfall (ES). Return, Sd, MDD, Part, TO and ES are given in percentage points. For the historical path, the performance measures are calculated using the daily returns resulting from the strategy. For the historical block-bootstrap, the performance measures are based on the simulated yearly returns, except for MDD, Calmar ratio and participation. Those are based on the daily risky asset exposure of the corresponding draw and show the yearly mean of the specific measure.

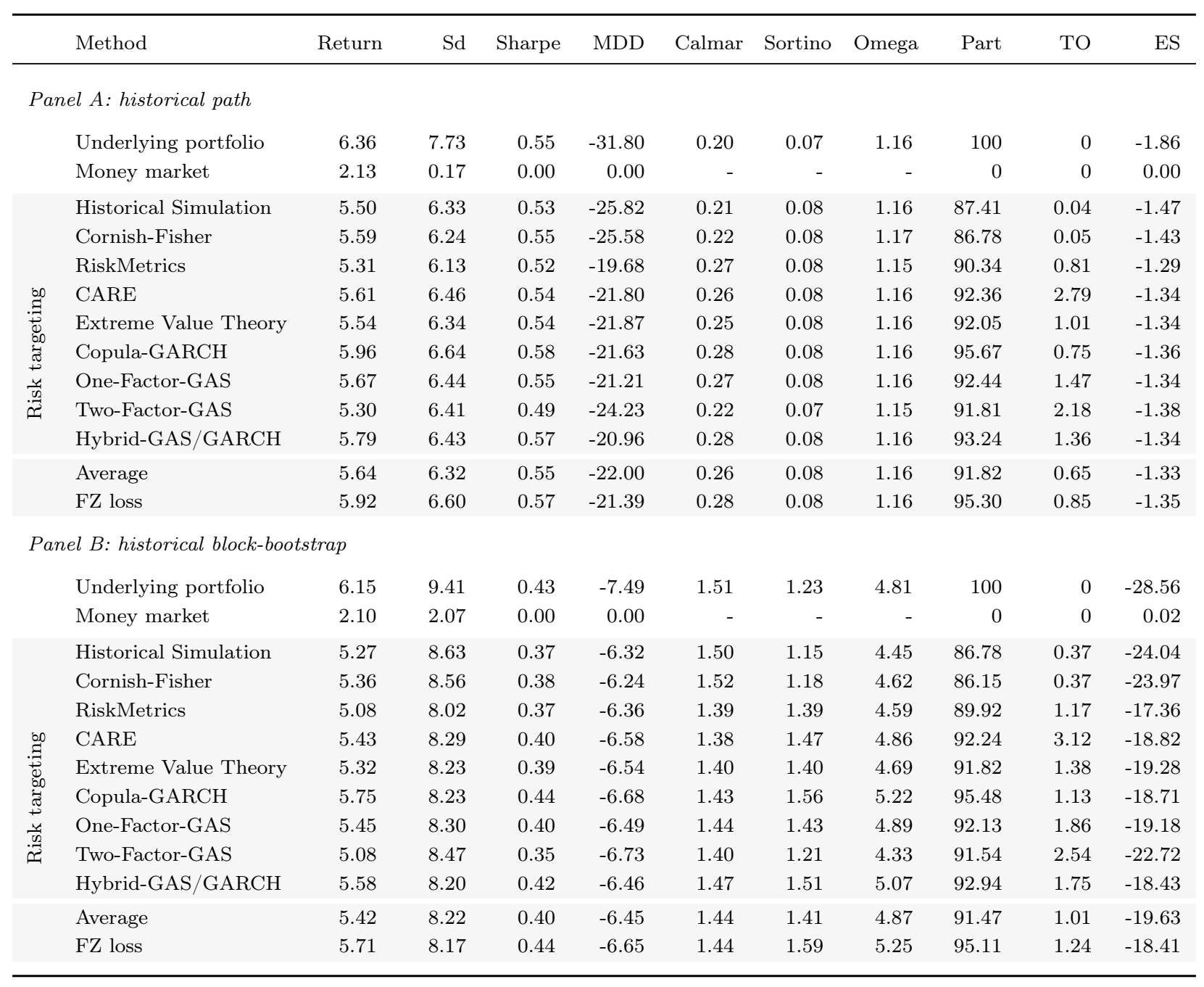


Table VI

\section{Risk Targeting: Various Portfolios and Target Levels}

This table shows the backtesting results of the risk targeting strategy based on different risk forecasts for the historical path and the historical block-bootstrap over the sample period 1996 to 2017 using various portfolios and various target levels. To benchmark the results of the ES targeting strategy based on the multi-asset portfolio we estimate the ES targeting strategy also for four different test portfolio allocations. We choose a pure equity portfolio (4\% ES target), a pure bond portfolio (1.5\% ES target), a 30/70 equity/bond portfolio (1.5\% ES target) and a 60/40 equity/bond portfolio (2.5\% ES target) as underlyings for the ES targeting strategy. Moreover, we check the robustness of the multi-asset results with respect to the chosen ES target level, here: $1 \%, 1.25 \%, 1.5 \%, 1.75 \%$ and $2 \%$. We base our comparison on the Calmar ratio for the historical path and on the Omega ratio for the historical block-bootstrap.

\begin{tabular}{|c|c|c|c|c|c|c|c|c|c|c|}
\hline & \multicolumn{5}{|c|}{ Portfolios } & \multicolumn{5}{|c|}{ Target levels } \\
\hline & Multi-Asset & Equity & Bond & $30-70$ & $60-40$ & $1 \%$ & $1.25 \%$ & $1.5 \%$ & $1.75 \%$ & $2 \%$ \\
\hline \multicolumn{11}{|c|}{ Panel A: historical path (Calmar ratio) } \\
\hline Historical Simulation & 0.21 & 0.20 & 0.58 & 0.32 & 0.19 & 0.25 & 0.23 & 0.21 & 0.20 & 0.20 \\
\hline Cornish-Fisher & 0.22 & 0.20 & 0.57 & 0.35 & 0.20 & 0.25 & 0.23 & 0.22 & 0.21 & 0.21 \\
\hline RiskMetrics & 0.27 & 0.21 & 0.66 & 0.36 & 0.22 & 0.30 & 0.28 & 0.27 & 0.27 & 0.27 \\
\hline CARE & 0.26 & 0.21 & 0.64 & 0.16 & 0.19 & 0.28 & 0.26 & 0.26 & 0.25 & 0.25 \\
\hline Extreme Value Theory & 0.25 & 0.20 & 0.64 & 0.33 & 0.23 & 0.28 & 0.26 & 0.25 & 0.25 & 0.25 \\
\hline Copula-GARCH & 0.28 & 0.20 & 0.64 & 0.37 & 0.23 & 0.31 & 0.29 & 0.28 & 0.27 & 0.25 \\
\hline One-Factor-GAS & 0.27 & 0.20 & 0.64 & 0.35 & 0.27 & 0.30 & 0.27 & 0.27 & 0.26 & 0.26 \\
\hline Two-Factor-GAS & 0.22 & 0.21 & 0.65 & 0.37 & 0.23 & 0.23 & 0.21 & 0.22 & 0.22 & 0.21 \\
\hline Hybrid-GAS/GARCH & 0.28 & 0.21 & 0.60 & 0.42 & 0.24 & 0.30 & 0.27 & 0.28 & 0.28 & 0.27 \\
\hline Average & 0.26 & 0.21 & 0.64 & 0.36 & 0.24 & 0.30 & 0.27 & 0.26 & 0.26 & 0.25 \\
\hline FZ loss & 0.28 & 0.20 & 0.64 & 0.36 & 0.23 & 0.33 & 0.29 & 0.28 & 0.27 & 0.25 \\
\hline \multicolumn{11}{|c|}{ Panel B: historical block-bootstrap (Omega ratio) } \\
\hline Historical Simulation & 4.45 & 2.13 & 25.29 & 12.04 & 3.86 & 5.62 & 4.88 & 4.45 & 4.29 & 4.33 \\
\hline Cornish-Fisher & 4.62 & 2.21 & 24.65 & 12.05 & 4.00 & 5.80 & 5.05 & 4.62 & 4.42 & 4.56 \\
\hline RiskMetrics & 4.59 & 2.47 & 24.43 & 12.17 & 4.74 & 4.77 & 4.33 & 4.59 & 5.15 & 5.32 \\
\hline CARE & 4.86 & 2.44 & 20.28 & 3.79 & 3.82 & 5.28 & 4.75 & 4.86 & 5.10 & 5.38 \\
\hline Extreme Value Theory & 4.69 & 2.27 & 23.70 & 11.57 & 4.31 & 5.15 & 4.70 & 4.69 & 4.98 & 5.28 \\
\hline Copula-GARCH & 5.22 & 2.31 & 24.96 & 14.41 & 4.49 & 4.95 & 4.75 & 5.22 & 5.39 & 5.36 \\
\hline One-Factor-GAS & 4.89 & 2.45 & 21.81 & 12.94 & 5.22 & 5.49 & 4.88 & 4.89 & 5.18 & 5.25 \\
\hline Two-Factor-GAS & 4.33 & 2.34 & 21.24 & 12.60 & 4.65 & 4.38 & 4.04 & 4.33 & 4.58 & 4.67 \\
\hline Hybrid-GAS/GARCH & 5.07 & 2.30 & 22.42 & 17.33 & 4.70 & 5.16 & 4.64 & 5.07 & 5.40 & 5.49 \\
\hline Average & 4.87 & 2.32 & 25.53 & 12.95 & 4.76 & 5.84 & 4.94 & 4.87 & 5.18 & 5.28 \\
\hline FZ loss & 5.25 & 2.39 & 23.86 & 13.06 & 4.36 & 5.74 & 5.02 & 5.25 & 5.49 & 5.46 \\
\hline
\end{tabular}


Table VII

\section{DPPI for Multi-Asset Portfolio}

This table shows the backtesting results of the multi-asset DPPI strategy with conditional multipliers based on different 1\%-ES forecasts for the historical path (Panel A) and the historical block-bootstrap (Panel B) over the sample period 1996 to 2017. For comparison, we include a static multiplier (CPPI) based on the maximum portfolio loss (resulting in $m=8)$ as well as the performance of the underlying multi-asset portfolio and the money market investment. In each calendar year, a floor of $95 \%$ of the initial portfolio value is installed. We report the annualized mean return (Return), annualized standard deviation (Sd), Sharpe ratio (SR), maximum drawdown (MDD), Calmar ratio, Sortino ratio, Omega ratio, participation in the risky multi-asset portfolio (Part), turnover (TO) and the 1\% Expected Shortfall (ES). Return, Sd, MDD, Part, TO and ES are given in percentage points. For the historical path, the performance measures are calculated using the daily returns resulting from the strategy. For the historical block-bootstrap, the performance measures are based on the simulated yearly returns, except for MDD, Calmar ratio and participation. Those are based on the daily risky asset exposure of the corresponding draw and show the yearly mean of the specific measure.

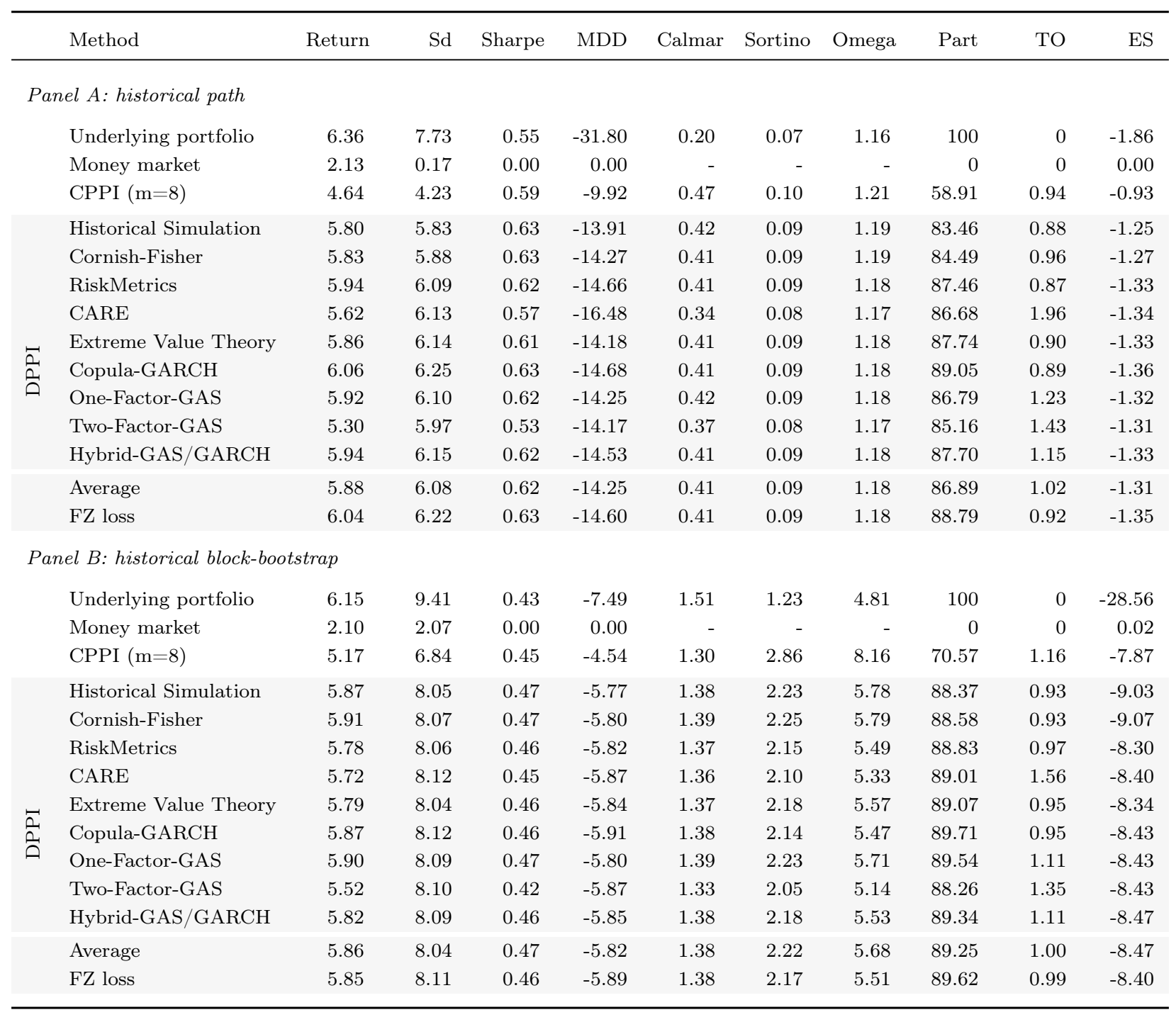




\section{Table VIII}

\section{DPPI: Various Portfolios and Floors}

This table shows the backtesting results of the DPPI strategy with conditional multipliers based on different risk forecasts for the historical path and the historical block-bootstrap over the sample period 1996 to 2017 using various portfolios and various floor levels. To benchmark the results of the DPPI strategy based on the multi-asset portfolio we also backtest the DPPI strategy for four different test portfolio allocations. To this end, we choose a pure equity portfolio ( $80 \%$ floor), a pure bond portfolio (95\% floor), a 30/70 equity/bond portfolio (95\% floor) and a $60 / 40$ equity/bond portfolio (90\% floor) as underlying for the DPPI strategy. Moreover, we check the robustness of the results of the multi-asset DPPI strategy with respect to the chosen floor level, here: $93 \%, 94 \%, 95 \%, 96 \%$, and $97 \%$. We base our comparison on the Calmar ratio for the historical path and on the Omega ratio for the historical block-bootstrap.

\begin{tabular}{|c|c|c|c|c|c|c|c|c|c|c|}
\hline & \multicolumn{5}{|c|}{ Portfolios } & \multicolumn{5}{|c|}{ Floors } \\
\hline & Multi-Asset & Equity & Bond & $30-70$ & $60-40$ & $93 \%$ & $94 \%$ & $95 \%$ & $96 \%$ & $97 \%$ \\
\hline \multicolumn{11}{|c|}{ Panel A: historical path (Calmar ratio) } \\
\hline Historical Simulation & 0.42 & 0.17 & 0.53 & 0.55 & 0.25 & 0.37 & 0.38 & 0.42 & 0.48 & 0.57 \\
\hline Cornish-Fisher & 0.41 & 0.17 & 0.51 & 0.57 & 0.26 & 0.37 & 0.38 & 0.41 & 0.47 & 0.54 \\
\hline RiskMetrics & 0.41 & 0.14 & 0.60 & 0.65 & 0.20 & 0.37 & 0.39 & 0.41 & 0.39 & 0.34 \\
\hline CARE & 0.34 & 0.19 & 0.51 & 0.49 & 0.24 & 0.36 & 0.36 & 0.34 & 0.37 & 0.39 \\
\hline Extreme Value Theory & 0.41 & 0.17 & 0.60 & 0.60 & 0.23 & 0.37 & 0.39 & 0.41 & 0.42 & 0.42 \\
\hline Copula-GARCH & 0.41 & 0.15 & 0.60 & 0.70 & 0.24 & 0.38 & 0.39 & 0.41 & 0.40 & 0.46 \\
\hline One-Factor-GAS & 0.42 & 0.17 & 0.57 & 0.60 & 0.24 & 0.38 & 0.39 & 0.42 & 0.45 & 0.49 \\
\hline Two-Factor-GAS & 0.37 & 0.16 & 0.57 & 0.54 & 0.23 & 0.34 & 0.36 & 0.37 & 0.37 & 0.35 \\
\hline Hybrid-GAS/GARCH & 0.41 & 0.16 & 0.56 & 0.59 & 0.24 & 0.38 & 0.39 & 0.41 & 0.42 & 0.43 \\
\hline Average & 0.41 & 0.17 & 0.59 & 0.61 & 0.25 & 0.37 & 0.39 & 0.41 & 0.44 & 0.43 \\
\hline FZ loss & 0.41 & 0.18 & 0.59 & 0.65 & 0.24 & 0.37 & 0.39 & 0.41 & 0.44 & 0.47 \\
\hline \multicolumn{11}{|c|}{ Panel B: historical block-bootstrap (Omega ratio) } \\
\hline Historical Simulation & 5.78 & 2.56 & 13.61 & 14.21 & 4.36 & 5.39 & 5.58 & 5.78 & 6.07 & 6.44 \\
\hline Cornish-Fisher & 5.79 & 2.58 & 13.26 & 14.54 & 4.47 & 5.41 & 5.59 & 5.79 & 6.06 & 6.44 \\
\hline RiskMetrics & 5.49 & 2.39 & 13.51 & 14.33 & 4.00 & 5.29 & 5.38 & 5.49 & 5.61 & 5.70 \\
\hline CARE & 5.33 & 2.87 & 9.88 & 9.70 & 4.18 & 5.29 & 5.31 & 5.33 & 5.38 & 5.39 \\
\hline Extreme Value Theory & 5.57 & 2.62 & 14.42 & 14.59 & 4.12 & 5.36 & 5.45 & 5.57 & 5.69 & 5.78 \\
\hline Copula-GARCH & 5.47 & 2.51 & 12.74 & 16.37 & 4.10 & 5.31 & 5.38 & 5.47 & 5.59 & 5.70 \\
\hline One-Factor-GAS & 5.71 & 2.85 & 12.43 & 15.19 & 4.71 & 5.52 & 5.64 & 5.71 & 5.80 & 5.93 \\
\hline Two-Factor-GAS & 5.14 & 2.71 & 11.33 & 12.72 & 4.57 & 5.21 & 5.20 & 5.14 & 5.09 & 5.13 \\
\hline Hybrid-GAS/GARCH & 5.53 & 2.69 & 12.00 & 15.69 & 4.72 & 5.37 & 5.45 & 5.53 & 5.68 & 5.86 \\
\hline Average & 5.68 & 2.67 & 13.27 & 14.84 & 4.40 & 5.41 & 5.53 & 5.68 & 5.84 & 5.98 \\
\hline FZ loss & 5.51 & 2.78 & 12.81 & 14.83 & 4.18 & 5.34 & 5.43 & 5.51 & 5.66 & 5.79 \\
\hline
\end{tabular}




\section{Figure 1. Standalone VaR and ES Forecasts over Time}

This figure shows the daily 1\%-VaR forecasts (in black) and associated ES forecasts (in blue) of the different standalone risk models as well as the realized returns of the multi-asset portfolio (grey dots) over the period 02/10/1996 to $31 / 3 / 2017$. VaR violations are marked in red. At a confidence level of $1 \%$, a total of 53 violations are expected over the model period.

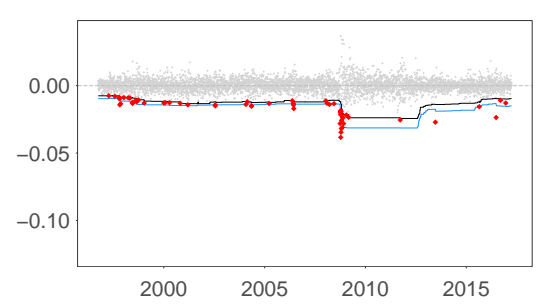

(a) Historical Simulation

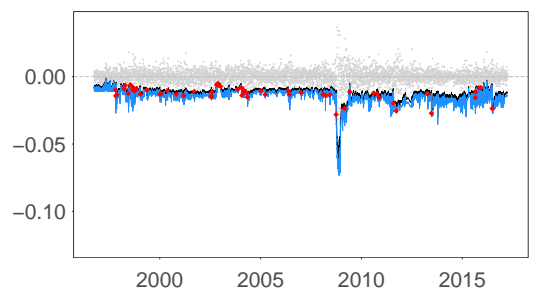

(d) CARE

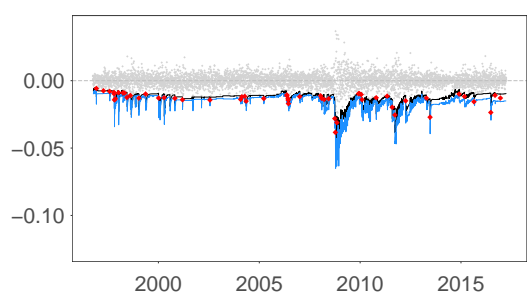

(g) One-factor GAS

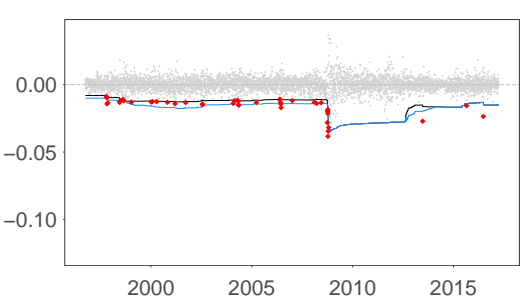

(b) Cornish-Fisher

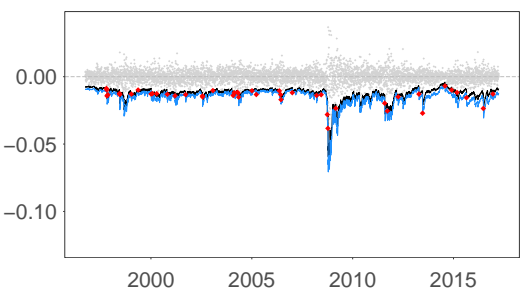

(e) EVT

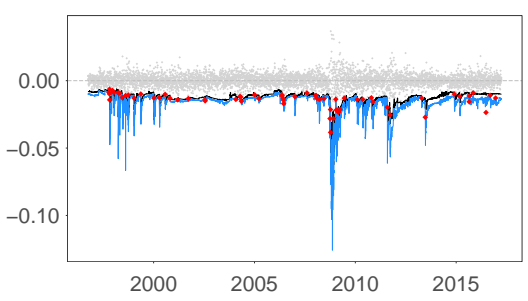

(h) Two-factor GAS

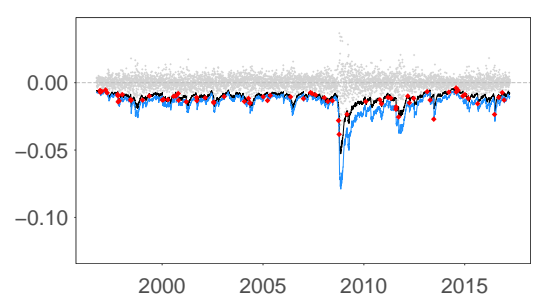

(c) RiskMetrics

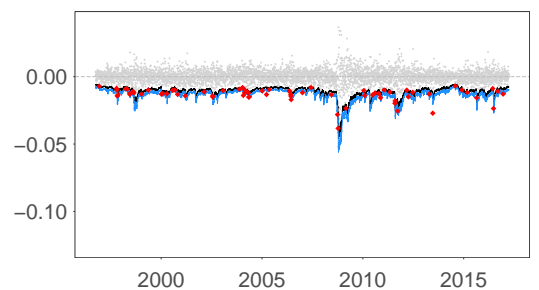

(f) Copula-GARCH

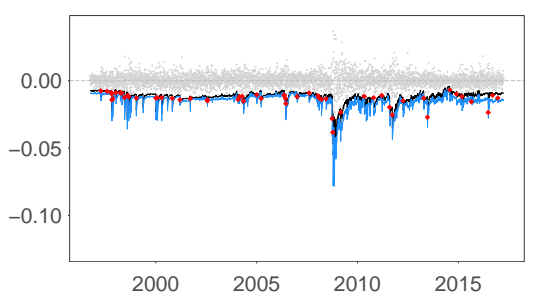

(i) Hybrid GAS/GARCH 


\section{Figure 2. Forecast Combination}

This figure illustrates the weights and forecasts of the FZ loss combination approach for the multi-asset portfolio. Panel (a) shows the estimated ES and VaR combination weights over the sample period from 1996 to 2017 . Panel (b) illustrates the mean of ES and VaR combination weights for all portfolios. Panel (c) and (d) show the daily $1 \%$-VaR forecasts (in black) and associated ES forecasts (in blue) from the FZ loss and the simple average combination approaches. Realized returns of the multi-asset portfolio and VaR violations are marked in grey and red, respectively.
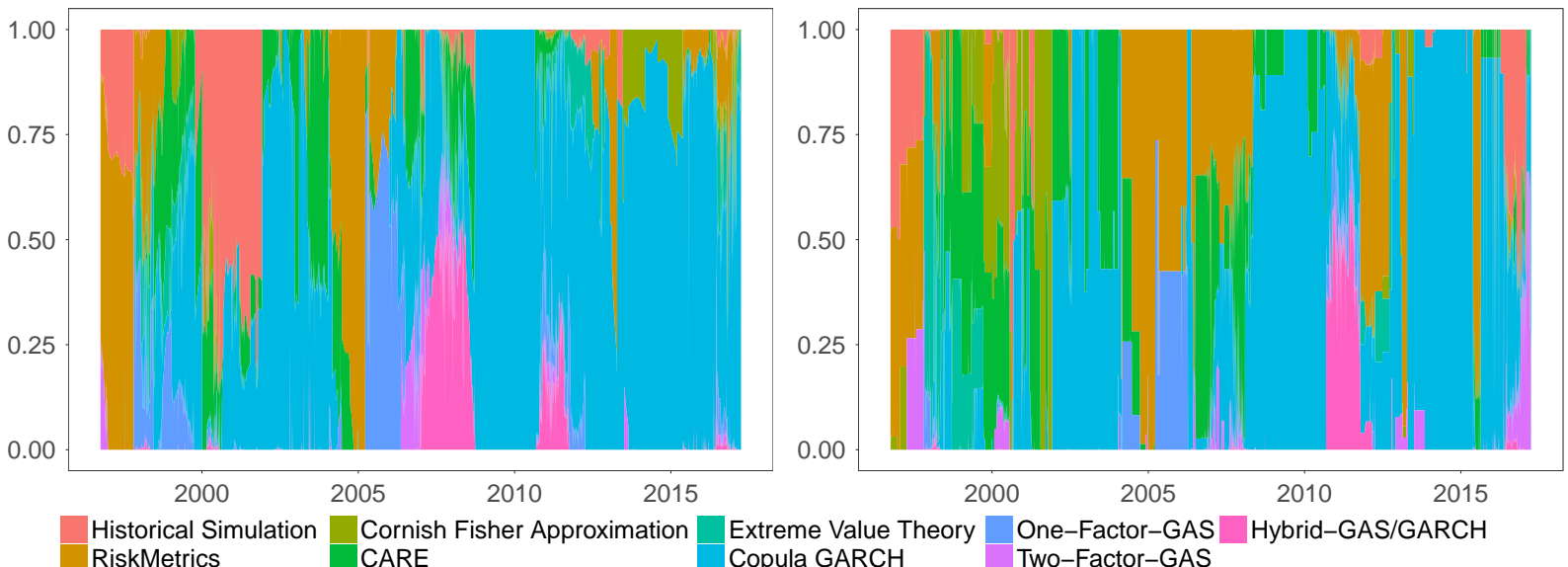

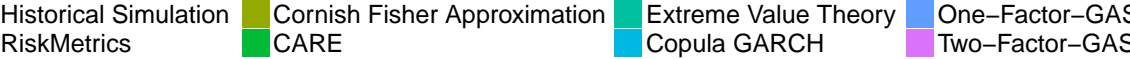

(a) ES and VaR combination weights over time

\begin{tabular}{|c|c|c|c|c|c|c|c|c|c|c|}
\hline 0.09 & 0.04 & 0.05 & 0.04 & 0.02 & Historical Simulation & 0.05 & 0.07 & 0.13 & 0.1 & 0.09 \\
\hline 0.13 & 0.14 & 0.23 & 0.14 & 0.15 & RiskMetrics & 0.2 & 0.16 & 0.28 & 0.2 & 0.13 \\
\hline 0.03 & 0.02 & 0.17 & 0.13 & 0.04 & Cornish Fisher Approximation & 0.06 & 0.03 & 0.06 & 0.02 & 0.01 \\
\hline 0.1 & 0.22 & 0.13 & 0.1 & 0.24 & CARE & 0.16 & 0.33 & 0.12 & 0.09 & 0.27 \\
\hline 0.03 & 0.14 & 0.05 & 0.03 & 0.06 & Extreme Value Theory & 0.04 & 0.08 & 0.03 & 0.01 & 0.03 \\
\hline 0.51 & 0.32 & 0.27 & 0.42 & 0.37 & Copula GARCH & 0.41 & 0.17 & 0.25 & 0.44 & 0.28 \\
\hline 0.06 & 0.03 & 0.01 & 0.03 & 0.06 & One-Factor-GAS & 0.04 & 0.08 & 0.01 & 0.03 & 0.09 \\
\hline 0.02 & 0.04 & 0.04 & 0.06 & 0.01 & Two-Factor-GAS & 0.02 & 0.03 & 0.05 & 0.03 & 0.04 \\
\hline 0.04 & 0.04 & 0.06 & 0.04 & 0.06 & Hybrid-GAS/GARCH & 0.03 & 0.06 & 0.07 & 0.08 & 0.05 \\
\hline
\end{tabular}

(b) ES and VaR mean combination weights

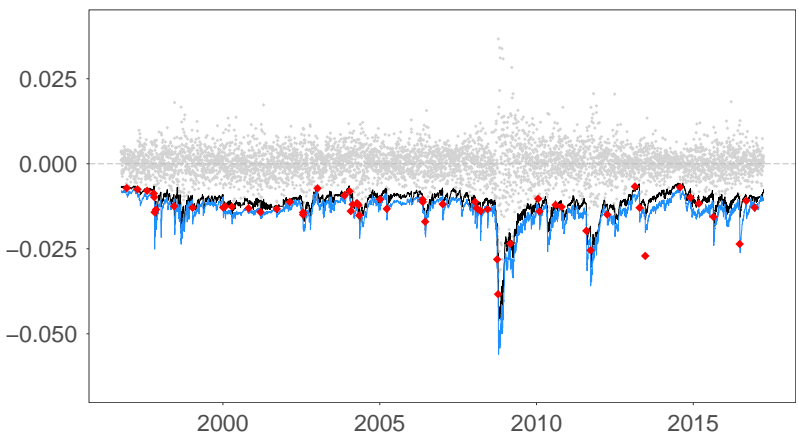

(c) FZ loss combination forecasts

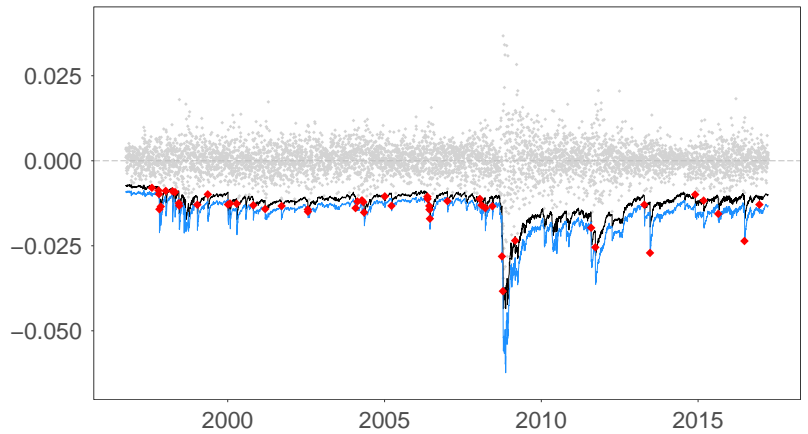

(d) Average combination forecasts 


\section{Figure 3. Historical Path and Historical Block-Bootstrap of Risk Targeting}

This chart illustrates the performance of the ES targeting strategy with multi-asset underlying portfolio (35\% equities, $40 \%$ fixed income, $15 \%$ commodities, $45 \%$ currencies). Panel (a) shows the historical path of the protected portfolio (red line) over the sample period 1996 to 2017. Exposure is calculated based on the 1\%-ES of the FZ loss combination approach. The target level is a 1.5\% ES. For comparison, we include the performance of the underlying multi-asset portfolio (blue line) and a money market investment (black line). Panel (b) shows the distribution of simulated yearly returns of the protected portfolio (red shading) and that of a buy-and-hold portfolio invested in the simulated multi-asset underlying (blue shading).

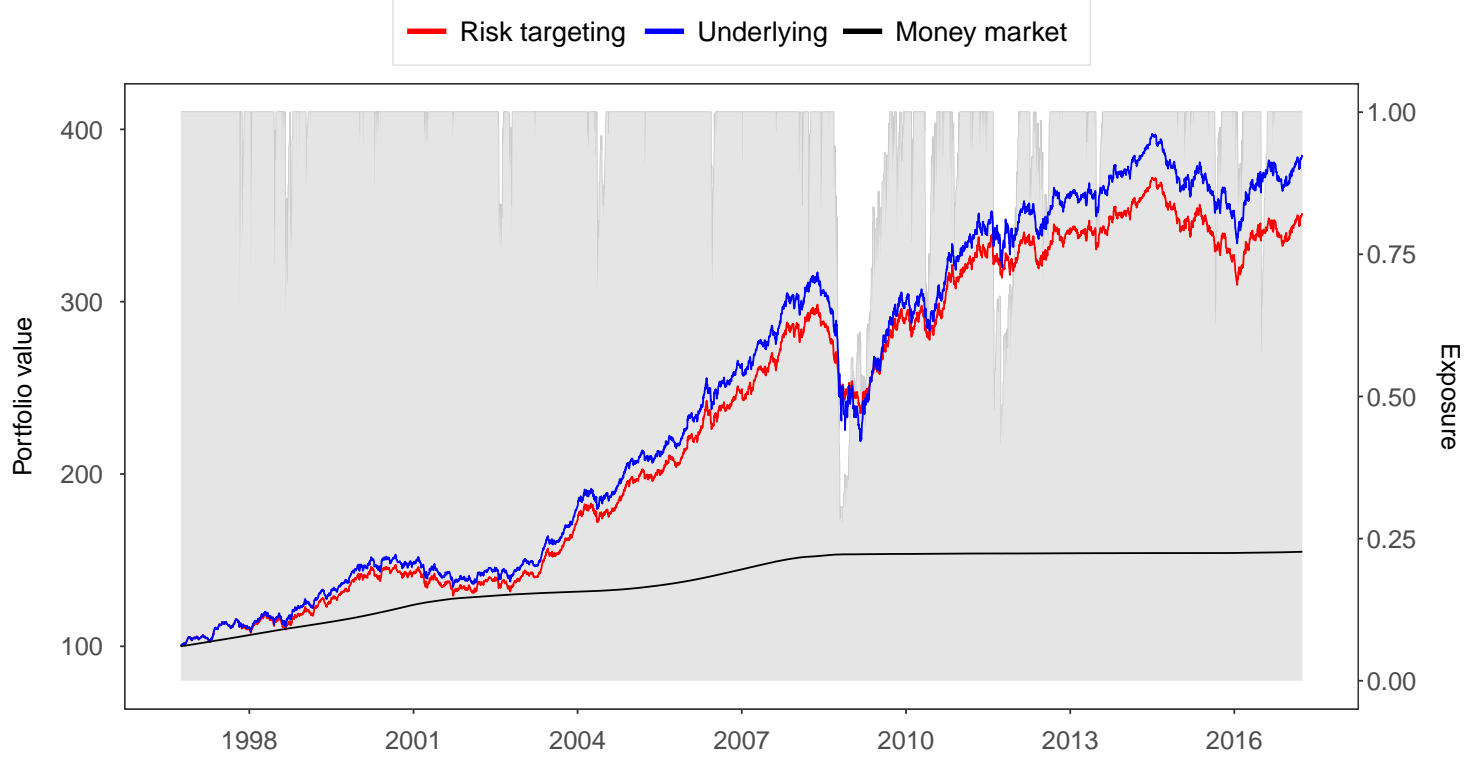

(a) Historical path

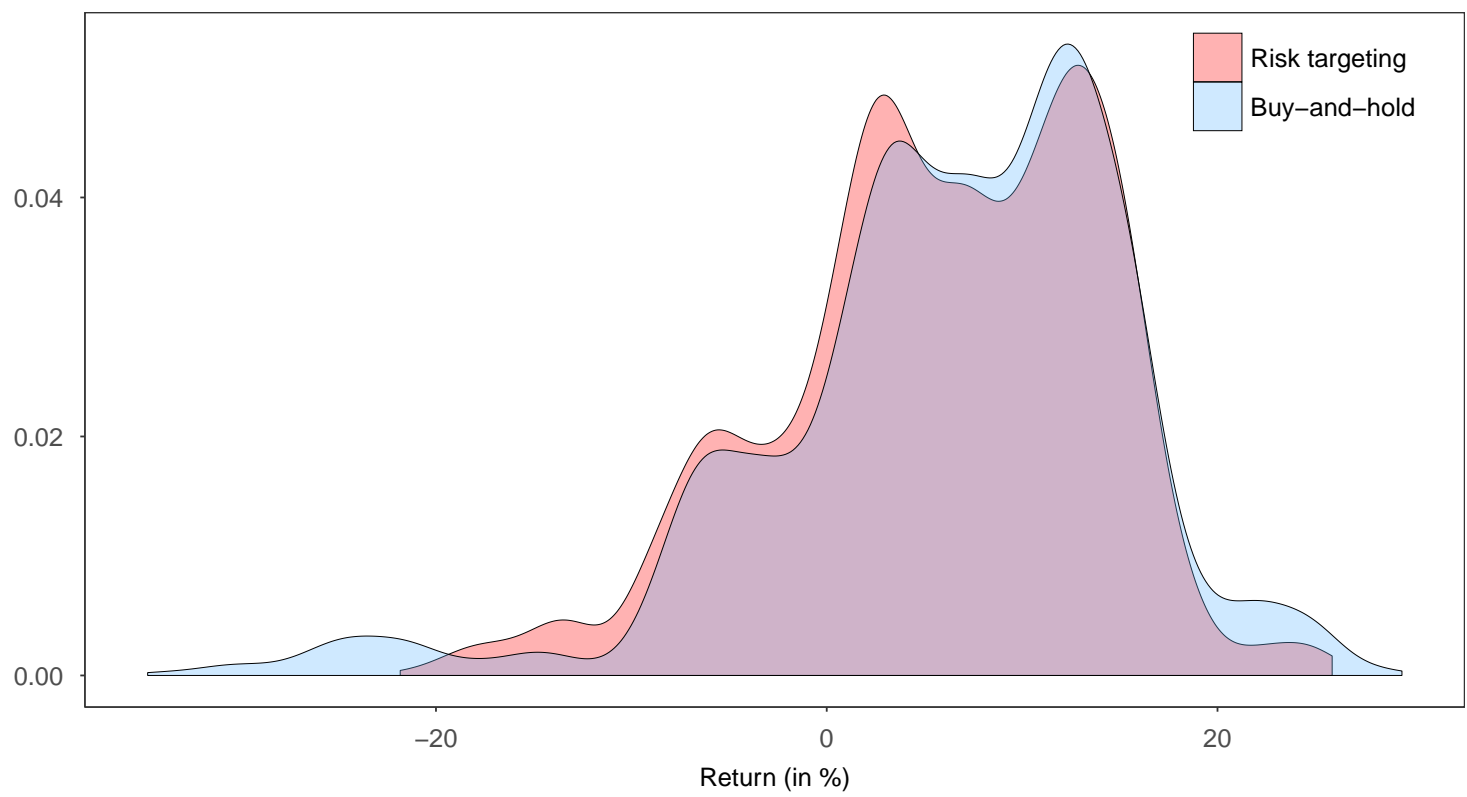

(b) Historical block-bootstrap 


\section{Figure 4. Historical Path and Historical Block-Bootstrap of DPPI}

This chart illustrates the performance of the DPPI strategy with multi-asset underlying portfolio (35\% equities, $40 \%$ fixed income, $15 \%$ commodities, $45 \%$ currencies). Panel (a) shows the historical path of the protected portfolio (red line) in relation to the floor (green line) over the sample period from 1996 to 2017. Exposure is calculated based on the 1\%-ES of the FZ loss combination approach. The floor level of the DPPI strategy is 95\%. For comparison, we include the performance of the underlying multi-asset portfolio (blue line) and a money market investment (black line). Panel (b) shows the distribution of simulated yearly returns of the protected portfolio (red shading) and that of a buy-and-hold portfolio invested in the simulated multi-asset underlying (blue shading).

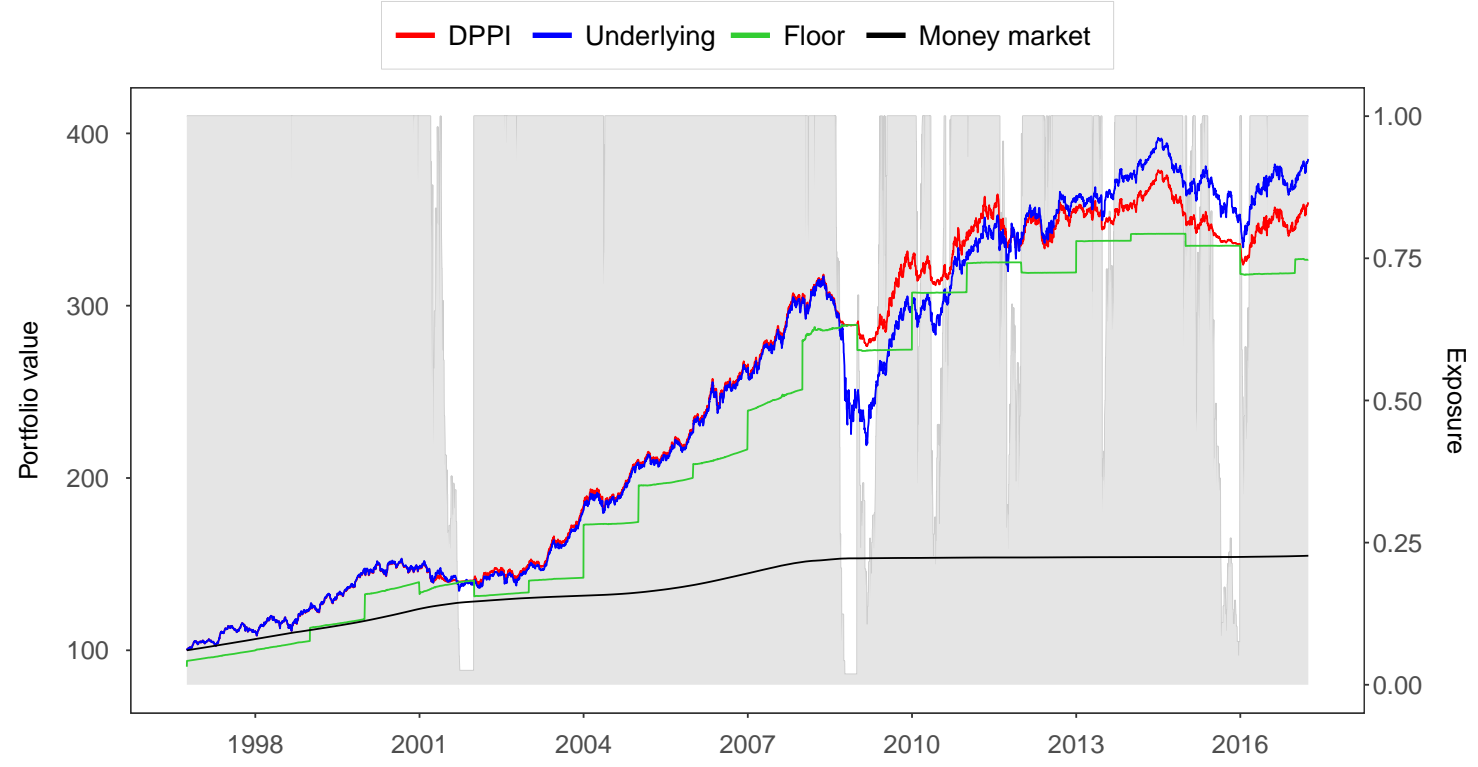

(a) Historical path

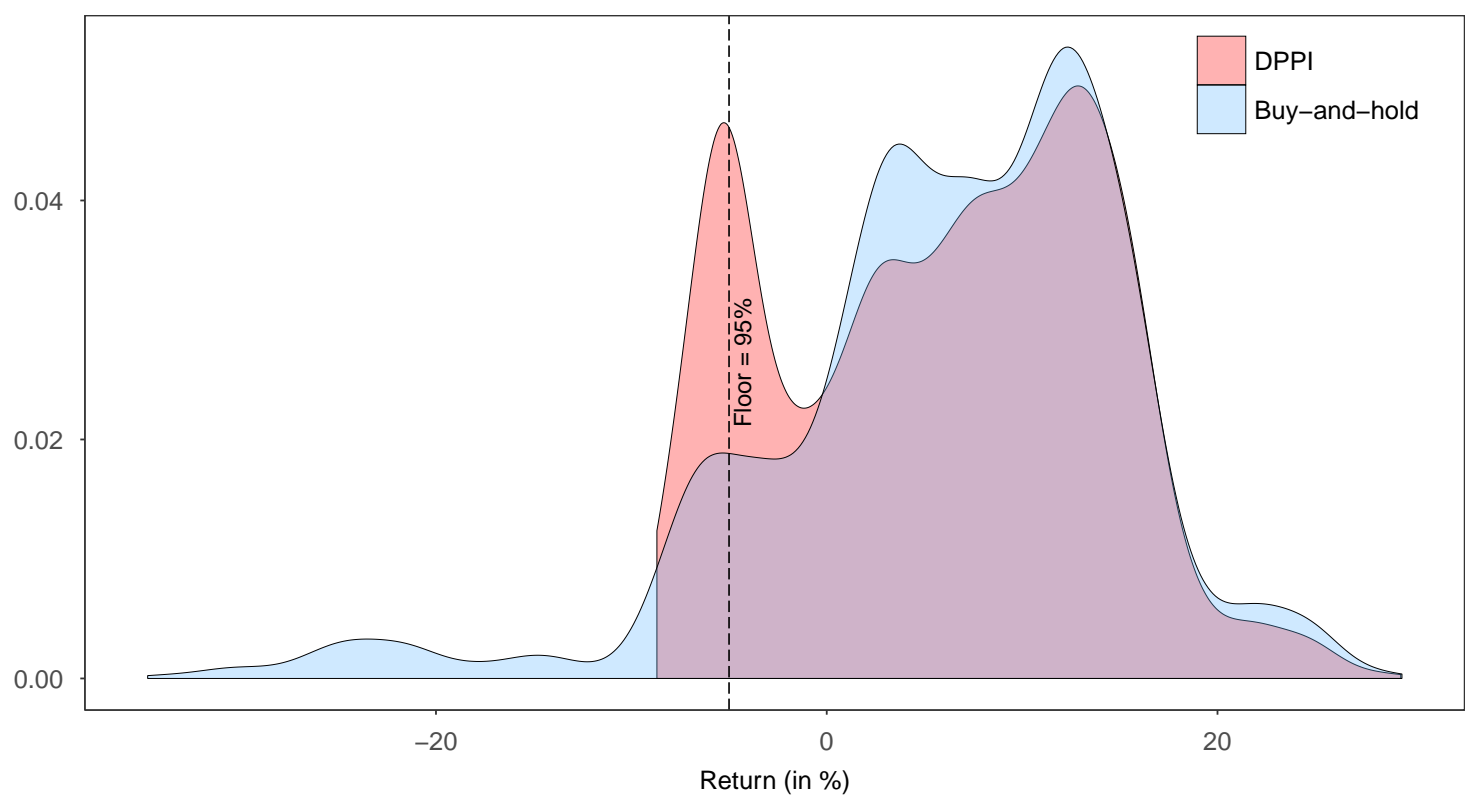

(b) Historical block-bootstrap 